\title{
EXISTENCE THEOREMS FOR WEAKLY SYMMETRIC OPERATIONS
}

\author{
MIKLÓS MARÓTI AND RALPH MCKENZIE
}

\section{INTRODUCTION}

A $k$-ary near-unanimity operation (or $k$-NU) on a set $A$ is an operation that satisfies the equations

$$
f(y, x, \ldots, x) \approx f(x, y, \ldots, x) \approx \cdots \approx f(x, x, \ldots, x, y) \approx x .
$$

A $k$-ary weak near-unanimity operation (or $k$-WNU) on $A$ is an operation that satisfies the equations

and

$$
w(x, \ldots x) \approx x
$$

$$
w(y, x, \ldots, x) \approx w(x, y, \ldots, x) \approx \cdots \approx w(x, x, \ldots, x, y) .
$$

If an algebra $\mathbf{A}$ has a $k$-NU (or a $k$-WNU) term operation, we say that $\mathbf{A}$ satisfies $\mathrm{NU}(k)$ (or $\mathrm{WNU}(k)$, respectively). Likewise, a variety is said to satisfy $\mathrm{NU}(k)$ (or $\mathrm{WNU}(k)$, respectively), it it has a $k$-variable term satisfying these equations.

It has been conjectured that a finite idempotent algebra $\mathbf{A}$ has finite relational width if and only if $\mathrm{V}(\mathbf{A})$ (the variety generated by $\mathbf{A}$ ) has meet semi-distributive congruence lattices. The concept of "finite relational width" arises in the theory of complexity of algorithms, in the algebraic study of constraint-satisfaction problems. Actually, there are several different definitions of this concept and it is not known if they are equivalent. One version of the concept and the conjecture mentioned above are due to B. Larose and L. Zádori [10].

The important family of varieties with meet semi-distributive congruence lattices has various known characterizations. There is a characterization by a certain Maltsev condition; also, it is known that a locally finite variety has this property iff it omits congruence covers of types $\mathbf{1}$ and $\mathbf{2}$ (defined in the tame congruence theory of D. Hobby, R. McKenzie [6]).

E. Kiss showed that a finite idempotent algebra of relational width $k$ must have an $m$-WNU term operation for every $m \geq k$. E. Kiss and M. Valeriote then observed that a finite algebra with a $k$-WNU term operation, $k>1$, must omit congruence covers of type $\mathbf{1}$. These observations led M. Valeriote to make two conjectures: any locally finite variety omits congruence covers of type $\mathbf{1}$ iff it satisfies WNU( $k$ ) for some $k>1$; any locally finite variety has meet semi-distributive congruence lattices if and only if for some $k$, it satisfies $\operatorname{WNU}(m)$ for all $m \geq k$. In this paper, we prove both of these conjectures of M. Valeriote.

The family of locally finite varieties omitting type $\mathbf{1}$ is the largest family of locally finite varieties defined by a nontrivial idempotent Maltsev condition. For this

Date: September 12, 2006.

The first author was partially supported by the Hungarian National Foundation for Scientific Research (OTKA), grant nos. T 48809 and T 60148. 
result, see D. Hobby, R. McKenzie [6], [Theorem 9.6]. Several equivalent characterizations for this largest Maltsev family are given in this theorem of D. Hobby and R. McKenzie. One that we will use states that a locally finite variety $\mathcal{V}$ omits type $\mathbf{1}$ if and only if $\mathcal{V}$ has a term $p(x, y, z)$ with the property that whenever $\mathbf{A}$ is an algebra in $\mathcal{V}$ and $\theta$ is a locally solvable congruence of $\mathbf{A}$ and $(a, b) \in \theta$, then $p(a, b, b)=a=p(b, b, a)$. An important corollary of this characterization, which we need, is that in such a variety, every Abelian algebra is polynomially equivalent to a module over a ring.

The result we prove in this paper, that a locally finite variety $\mathcal{V}$ omits type 1 iff $\mathcal{V} \models \mathrm{WNU}(k)$ for some $k>1$, is the simplest known Maltsev characterization of this family of varieties.

In addition to proving the two conjectures of M. Valeriote, we shall demonstrate here that every congruence distributive variety satisfies $\mathrm{WNU}(k)$ for all $k \geq 3$. (This result requires no assumption of local finiteness.) Thus, in particular, it follows that $\mathrm{NU}(n)$ implies $\mathrm{WNU}(k)$ whenever $k \geq 3$ and $n \geq 3$. A consequence of our characterization of locally finite varieties omitting type $\mathbf{1}$ is that every locally finite, congruence modular variety must satisfy $\mathrm{WNU}(k)$ for some $k>1$. Our final contribution will be a second proof of this fact about congruence modular varieties.

The chief results of this paper are listed below. The remainder of the paper is devoted to presenting proofs of these results.

Theorem 1.1. Let $\mathcal{V}$ be a locally finite variety. The following are equivalent:

(1) $\mathcal{V}$ omits type $\mathbf{1 .}$

(2) There is an integer $k>1$ such that $\mathcal{V} \models \mathrm{WNU}(k)$.

(3) There is an integer $n>1$ such that $\mathcal{V} \mid=\mathrm{WNU}(k)$ for all $k>1$ with $k \equiv 1$ $(\bmod n)$

Theorem 1.2. Let $\mathcal{V}$ be a locally finite variety. The following are equivalent:

(1) $\mathcal{V}$ omits types $\mathbf{1}$ and $\mathbf{2}$.

(2) The congruence lattices of all algebras in $\mathcal{V}$ are meet semi-distributive.

(3) There is an integer $m>1$ such that $\mathcal{V} \mid=\mathrm{WNU}(k)$ for all $k \geq m$.

(4) For every positive integer $n$ there is a positive integer $m$ such that $\mathcal{V} \models$ $\mathrm{WNU}(k)$ for all $k$ with $m+1 \leq k \leq m+n$.

Theorem 1.3. Every congruence distributive variety satisfies $\mathrm{WNU}(k)$ for all integers $k \geq 3$.

Corollary 1.4. Let $\mathcal{V}$ be a locally finite, congruence modular variety. There is an integer $n>1$ such that $\mathcal{V}=\mathrm{WNU}(k)$ for all $k>1$ with $k \equiv 1(\bmod n)$.

These results will be proved in the order listed. Our proof of Theorem 1.3 has a different character from our proofs of the first two theorems. The Corollary is a corollary to Theorem 1.1. However, as we mentioned, we shall present, in the final section of the paper, a different proof of Corollary 1.4 along the lines of our proof of Theorem 1.3.

\section{EASY OBSERVATIONS AND EXAMPLES}

It is well known that if a locally finite variety $\mathcal{V}$ admits type $\mathbf{1}$ then $\mathcal{V}$ cannot cannot satisfy any non-trivial idempotent Maltsev condition. In particular, it cannot have a weak near-unanimity term. This supplies one of two implications needed to prove Theorem 1.1. We shall now review the argument that proves this well-known 
fact, and review a closely related argument that reveals some limitations on the existence of weak near-unanimity terms that occur when $\mathcal{V}$ admits type $\mathbf{2}$. For the concepts and results used in these arguments, see D. Hobby, R. McKenzie [6].

Suppose that $\mathcal{V}$ has a type $\mathbf{1}$ congruence quotient. Then there is a finite algebra $\mathbf{F}$ in $\mathcal{V}$ and a minimal congruence $\mu$ in $\mathbf{F}$ such that $\left(0_{F}, \mu\right)$ has type $\mathbf{1}$. Let $U$ be a $\left(0_{F}, \mu\right)$-minimal set and $N$ be a $\left(0_{F}, \mu\right)$-trace contained in $U$ and $e(x)$ be a polynomial operation of $\mathbf{F}$ satisfying $e(e(x))=e(x)$ (for all $x \in F$ ) and $e(F)=U$. Suppose that $\mathbf{F}$ has a $k$-WNU-term $\tau(\bar{x})$. Then the operation $e(\tau(\bar{x}))$ restricted to $N$ is an operation on the set $N$ that satisfies the $\mathrm{WNU}(k)$-equations. This operation is a polynomial of the algebra $\left.\mathbf{F}\right|_{N}$. But since the type is $\mathbf{1}$, every polynomial operation of $\left.\mathbf{F}\right|_{N}$ depends on at most one of its variables. Hence $\left.\mathbf{F}\right|_{N}$ does not satisfy $\mathrm{WNU}(k)$ for any $k>1$, implying that $\mathbf{F}$, likewise does not satisfy $\mathrm{WNU}(k)$ for any $k>1$.

Next, we show that if $\mathcal{V}$ admits type $\mathbf{2}$ then there is a prime integer $p$ such that whenever $p$ divides $k$ then $\mathcal{V}$ admits no $k$-WNU term. This, and the observation above about type $\mathbf{1}$ quotients, give one of two implications needed to prove Theorem 1.2. Suppose that $\mathcal{V}$ has a type $\mathbf{2}$ congruence quotient. Choose a finite algebra $\mathbf{F}$ in $\mathcal{V}$ with a minimal congruence $\mu$ in $\mathbf{F}$ such that $\left(0_{F}, \mu\right)$ has type $\mathbf{2}$. Let $U$ be a $\left(0_{F}, \mu\right)$-minimal set and $N$ be a $\left(0_{F}, \mu\right)$-trace contained in $U$. In this situation, $\left.\mathbf{F}\right|_{N}$ is polynomially equivalent to a one-dimensional vector space over a finite field $\mathbf{k}$. Let $p$ be the characteristic of $\mathbf{k}$. It is easily verified that if $\left.\mathbf{F}\right|_{N}$ has a $k$-WNU operation among its polynomial operations, then this operation is unique, and defined as

$$
\tau\left(x_{1}, \ldots, x_{k}\right)=r\left(x_{1}+\cdots+x_{k}\right),
$$

where $r \in \mathbf{k}$ and $k r=1$ in $\mathbf{k}$. Such $r$ exists in $\mathbf{k}$ iff $p$ does not divide $k$. Now the same argument as above shows that $\mathbf{F}$ cannot satisfy $\mathrm{WNU}(k)$ if $p$ divides $k$.

Example 2.1. Let $\mathbf{A}_{n}=\left\langle Z_{n}, x-y+z\right\rangle$ where $\left\langle Z_{n}, x+y\right\rangle$ is the group of integers modulo $n$. The term operations of this algebra are simply those operations which can be expressed in the form

$$
f\left(x_{0}, \ldots, x_{k-1}\right)=\sum_{i} k_{i} x_{i}
$$

where $k_{i}$ are integers and their sum is 1 modulo $n$. If this operation is a $k$-WNU, then all the $k_{i}$ are congruent modulo $n$. Thus $\mathbf{A}_{n} \models \mathrm{WNU}(k)$ iff $(k, n)=1$. For example, $\mathbf{A}_{6}$ satisfies $\mathrm{WNU}(5)$ but not $\mathrm{WNU}(2)$, WNU(3) nor $\mathrm{WNU}(4)$.

Let $\mathbf{A}=\langle Z, x-y+z\rangle$ where $\langle Z, x+y\rangle$ is the group of integers. This algebra does not satisfy $\mathrm{WNU}(k)$ for any $k \geq 3$.

Finally, we remark that if $\mathbf{A}$ is an Abelian algebra in a locally finite variety $\mathcal{V}$ that omits type $\mathbf{1}$, then $\mathbf{A}$ does have a $k$-WNU for some $k>1$. In fact, by D. Hobby, R. McKenzie [6] Theorem 9.6, $\mathbf{A}$ has a term operation $p(x, y, z)$ satisfying Maltsev's equations $p(x, y, y)=x$ and $p(x, x, y)=y$. It is well known (see [4]) that every Abelian algebra $\mathbf{A}$ with such an operation is polynomially equivalent to a unitary module; and that all term operations of the module of the form $f\left(x_{0}, \ldots, x_{k-1}\right)=$ $\sum_{i} k_{i} x_{i}$, where $k_{i}$ are integers and their sum is 1 , are term operations of $\mathbf{A}$. Hence A does have $k$-WNU term operations in this case (in fact, for every $k>1$ that is relatively prime to the cardinality of $\mathbf{A}$ ). 


\section{Reduction to idempotent Algebras}

An operation $f\left(x_{1}, \ldots, x_{n}\right)$ on a set $A$ is said to be idempotent if $f(a, \ldots, a)=a$ for all $a \in A$. An algebra $\mathbf{A}=\langle A, \cdots\rangle$ is called idempotent iff all the operations of $\mathbf{A}$ are idempotent, equivalently, every one-element subset of $A$ is a subalgebra of $\mathbf{A}$. By an idempotent term of a variety $\mathcal{V}$ we mean a term for which $t(x, x, \ldots, x) \approx x$ is a law of $\mathcal{V}$. A variety is called idempotent if all of its algebras are idempotent, equivalently, if for all terms of $\mathcal{V}$ are idempotent.

If $\mathcal{V}$ is any variety, there is the idempotent reduct of $\mathcal{V}$, or $\mathcal{V}^{\text {id }}$. The variety $\mathcal{V}^{\text {id }}$ is idempotent. It is formed in this way: For every idempotent term $t=t(\bar{x})$ of $\mathcal{V}$, there is a corresponding basic operation $f_{t}(\bar{x})$ of $\mathcal{V}^{\text {id }}$ with the same number of variables as $t$. The signature of $\mathcal{V}^{\text {id }}$ consists just of the operation symbols $f_{t}$ with $t$ ranging over the idempotent term operations of $\mathcal{V}$. For every algebra $\mathbf{A} \in \mathcal{V}$ there is the algebra $\mathbf{A}^{\mathrm{id}}$ of the same signature as $\mathcal{V}^{\mathrm{id}}$,

$$
\mathbf{A}^{\mathrm{id}}=\left\langle A,\left\{t^{\mathbf{A}}: t \text { an idempotent term of } \mathcal{V}\right\}\right\rangle
$$

whose basic operations are the term operations over $A$ induced by the terms that are idempotent over $\mathcal{V}$. Then $\mathcal{V}^{\text {id }}$ is defined as the variety generated by all the algebras $\mathbf{A}^{\text {id }}$, with $\mathbf{A}$ ranging over $\mathcal{V}$. It can be shown that for any positive integer $n$, the free algebra of rank $n$ in $\mathcal{V}^{\text {id }}$, or $\mathbf{F}_{\mathcal{V}^{\text {id }}}(n)$, is isomorphic over the free generators, to the subalgebra of $\mathbf{F}_{\mathcal{V}}(n)^{\mathrm{id}}$ generated by the free generators of $\mathbf{F}_{\mathcal{V}}(n)$.

We mention this construction because it is an (easily verified) fact that each of the properties of algebras and of varieties that we are concerned with in this paper is invariant under the constructions $\mathbf{A} \mapsto \mathbf{A}^{\text {id }}, \mathcal{V} \mapsto \mathcal{V}^{\text {id }}$. An algebra $\mathbf{A}$ satisfies $\mathrm{WNU}(k)$ iff $\mathbf{A}^{\text {id }}$ satisfies $\mathrm{WNU}(k)$. The same is true of a variety $\mathcal{V}$. We have that $\mathcal{V}$ is congruence distributive, or congruence modular, iff $\mathcal{V}^{\text {id }}$ has the respective property. Moreover, if $\mathcal{V}$ is locally finite then $\mathcal{V}^{\text {id }}$ is locally finite; and in case $\mathcal{V}$ is locally finite, then $\mathcal{V}$ omits type $\mathbf{1}$ (respectively type $\mathbf{2}$ ) iff $\mathcal{V}^{\text {id }}$ omits type $\mathbf{1}$ (respectively type $\mathbf{2}$ ). (This is not obvious, but is a consequence of the Maltsev characterizations of these properties given in D. Hobby, R. McKenzie [6], Chapter 9.)

Thus both the hypotheses, and the conclusions, in each of our chief results, is invariant under passing to the idempotent reduct. Consequently, it will suffice to prove our results for idempotent varieties. Henceforth, we work only with idempotent algebras, and idempotent varieties. Idempotent algebras have several special properties that will be useful in our proofs. We mention that if $\theta$ is a congruence on an idempotent algebra $\mathbf{A}$, then every block of $\theta$ is a subalgebra of $\mathbf{A}$. Other useful properties will be explained as the need for them arises.

\section{Totally Symmetric RELATiOns}

From here to the end of this paper, all algebras are assumed to be idempotent, unless explicitly stated otherwise.

Definition 4.1. Let $\mathbf{A}$ be any algebra. A subuniverse $B$ of $\mathbf{A}^{n}$ is totally symmetric iff $B$ is invariant under all permutations of the coordinates. If $B$ is totally symmetric, then $B$ is a subdirect power of a certain subuniverse of $\mathbf{A}$, which we term $D(B)$ and call the domain of $B$. The projection of $B$ at each coordinate is equal to $D(B)$. 
To simplify notation, we shall write elements of $\mathbf{A}^{n}$ as though they were semigroup words. Thus, for example, $b a^{n-1}$ will denote the vector $(b, a, \ldots, a)$ in $A^{n}$ where $\{a, b\} \subseteq A$.

Definition 4.2. An algebra $\mathbf{B} \leq \mathbf{A}^{n}$ will be called a $\star$-subalgebra of $\mathbf{A}^{n}$ iff for some $\{a, b\} \subseteq A, \mathbf{B}$ is generated by the $n$ vectors $b a^{n-1}, a b a^{n-2}, \ldots, a^{n-1} b$. Note that in this case $B$ (the universe of $\mathbf{B}$ ) is totally symmetric, and $D(B)$ is generated by $\{a, b\}$.

Definition 4.3. Let $\mathbf{A}$ be an algebra and $k>1$ be an integer. We say that A satisfies $\mathrm{WNU}(k)$ iff $\mathbf{A}$ has a $k$-ary term operation $w(\bar{x})$ satisfying the weak near-unanimity equations

$$
w(y, x, \ldots, x) \approx w(x, y, x, \ldots, x) \approx \cdots \approx w(x, x, \ldots, x, y)
$$

(and $w(x, \ldots, x) \approx x$, but the idempotent equation is already assumed).

We say that $\mathbf{A}$ satisfies $\operatorname{ST}(k)$ iff every $\star$-subalgebra of $\mathbf{A}^{k}$ contains a diagonal vector $a^{k}$. We say that $\mathbf{A}$ satisfies $\operatorname{TS}(k)$ iff every non-empty totally symmetric subuniverse of $\mathbf{A}^{k}$ contains a diagonal vector.

We begin a sequence of lemmas detailing useful properties of these concepts.

Lemma 4.4. For a fixed signature and a fixed integer $k>1$, the class of algebras of the given signature satisfying TS(k) is closed under subalgebras, homomorphic images, and finite products.

Proof. It is straightforward to show that this class is closed under subalgebras and homomorphic images, and we leave this task to the reader. Now suppose that $\mathbf{C}=\mathbf{A} \times \mathbf{B}$ and that $\mathbf{A}=\operatorname{TS}(k)$ and $\mathbf{B}=\operatorname{TS}(k)$. Let $S$ be any non-empty totally symmetric subuniverse of $\mathbf{C}^{k}$. Let $\pi_{0}: \mathbf{C} \rightarrow \mathbf{A}$ be the first projection homomorphism. Then $\pi_{0}^{k}(S)=S_{0}$ is a non-empty totally symmetric subuniverse of $\mathbf{A}^{k}$. We can choose a diagonal element $a^{k}$ in $S_{0}$. Then

$$
S_{1}=\left\{\left(b_{0}, \ldots, b_{k-1}\right) \in B^{k}:\left(\left(a, b_{0}\right), \ldots,\left(a, b_{k-1}\right)\right) \in S\right\}
$$

is a non-empty totally symmetric subuniverse of $\mathbf{B}^{k}$. (Here, the idempotency of $\mathbf{A}$ ensures that $S_{1}$ is a subuniverse.) So there is $b \in B$ with $b^{k} \in S_{1}$. This means that $c^{k} \in S$, where $c=(a, b)$.

Lemma 4.5. Let $k>1$ be a positive integer.

(i) For any algebra $\mathbf{A}$, the implications $\mathrm{WNU}(k) \Rightarrow \mathrm{ST}(k)$ and $\mathrm{TS}(k) \Rightarrow \mathrm{ST}(k)$ are valid.

(ii) For any algebra $\mathbf{A}, \mathbf{A} \models \mathrm{WNU}(k)$ iff $\mathbf{A}$ belongs to some variety $\mathcal{V}$ such that the free algebra on two generators in $\mathcal{V}$ satisfies $\mathrm{ST}(k)$.

(iii) For any finite algebra $\mathbf{A}$, the implications $\mathrm{TS}(k) \Rightarrow \mathrm{WNU}(k) \Rightarrow \mathrm{ST}(k)$ are valid.

Proof. We regard (i) as obvious. Just note that if $w(\bar{x})$ is a $k$-variable weak-nu term for $\mathbf{A}$, and if $\mathbf{B} \subseteq \mathbf{A}^{k}$ is the subalgebra generated by the sequence of vectors (for some $\{a, b\} \subseteq A) \bar{q}_{i}=a^{i} b a^{k-i-1}, 0 \leq i<k$, then $w\left(\bar{q}_{0}, \ldots, \bar{q}_{k-1}\right)$ (with $w$ applied in $\mathbf{B})$ is the vector $c^{k}$ where $c=w(b, a, \ldots, a)$.

We remark that the truth of (ii) requires the assumed idempotency of algebras. To prove (ii), assume first that $\mathbf{A}$ satisfies $\mathrm{WNU}(k)$ and that $w(\bar{x})$ is a $k$-variable 
weak-nu term for $\mathbf{A}$. Let $\mathcal{V}$ be the variety generated by $\mathbf{A}$. Then $w(\bar{x})$ is a weak-nu term for every algebra in $\mathcal{V}$ (since the weak-nu property is determined by satisfaction of certain equations). Let, then, $\mathbf{F}$ be the free algebra on two generators in $\mathcal{V}$. By (i), $\mathbf{F} \models \mathrm{ST}(k)$, since $\mathbf{F} \models W N U(k)$. Now conversely, suppose that $\mathbf{F}$ is the free algebra freely generated by a two-element set $\{x, y\}$ in a (idempotent) variety $\mathcal{V}$, and that $\mathbf{F} \models S T(k)$. Let $\Delta_{k}$ be the subalgebra of $\mathbf{F}^{k}$ generated by the sequence of vectors $\bar{f}_{i}=x^{i} y x^{k-i-1}, 0 \leq i<k$. Since $\mathbf{F}=S T(k)$, there is a diagonal element $(c, \ldots, c)$ in $\Delta_{k}$. For some term $w\left(x_{0}, \ldots, x_{k-1}\right)$, we can write

$$
(c, \ldots, c)=w\left(\bar{f}_{0}, \bar{f}_{1}, \ldots, \bar{f}_{k-1}\right) .
$$

This means that for each $0 \leq i<k$,

$$
c=w\left(\bar{f}_{0}(i), \bar{f}_{1}(i), \ldots, \bar{f}_{k-1}(i)\right)=w(x, x, \ldots, x, y, x, \ldots, x)
$$

in which the final application of $w$ is to a sequence of $i x$ 's followed by $y$ and then $k-i-1 x$ 's. Since also $w(x, x, \ldots, x)=x$, then all the equations required for $w(\bar{x})$ to be a weak-nu term for $\mathcal{V}$ are satisfied. Thus $w(\bar{x})$ is a weak-nu term for every algebra that belongs to $\mathcal{V}$.

We now prove (iii). Let $\mathbf{A}$ be a finite algebra. All that remains is to show that $\mathbf{A} \models \operatorname{TS}(k)$ implies $\mathbf{A} \models \mathrm{WNU}(k)$. So let us assume that $\mathbf{A}$ satisfies $\mathrm{TS}(k)$. Let $\mathbf{F}$ be the free algebra on two generators in the variety generated by $\mathbf{A}$. Now $\mathbf{F}$ is finite, and is isomorphic to a subalgebra of a finite direct power of $\mathbf{A}$. By Lemma 4.4, $\mathbf{F} \models \mathrm{TS}(k)$; a fortiori, $\mathbf{F} \models \mathrm{ST}(k)$. Finally, by (ii) of this lemma, $\mathbf{A} \models \mathrm{WNU}(k)$.

The next three lemmas establish that if a finite algebra satisfies $\mathrm{WNU}(k)$ then it will satisfy $\operatorname{TS}\left(k^{\prime}\right)$ for some $k^{\prime} \geq k$.

Definition 4.6. By a special weak near-unanimity operation on a set $A$ we shall mean a weak-nu operation $w(\bar{x})$ on $A$ that satisfies the equation

$$
w(w(y, x, \ldots, x), x, \ldots, x) \approx w(y, x, \ldots, x) .
$$

Lemma 4.7. If a finite algebra of size $n$ has a weak near-unanimity term of arity $k$, then it has a special weak near-unanimity term of arity $k^{n !}$.

Proof. Let $\mathbf{A}$ be a finite algebra of size $n$ and $t$ be a weak near-unanimity term for $\mathbf{A}$ of arity $k$. We define by induction a sequence $t_{1}, t_{2}, \ldots, t_{n}$ ! of weak near-unanimity terms of arities $k, k^{2}, \ldots, k^{n !}$, respectively. Put $t_{1}=t$, and for all integers $i<n$ ! define

$$
\begin{aligned}
& t_{i+1}\left(x_{1}, \ldots, x_{k^{i+1}}\right) \\
& \quad=t_{i}\left(t\left(x_{1}, \ldots, x_{k}\right), t\left(x_{k+1}, \ldots, x_{2 k}\right), \ldots, t\left(x_{k\left(k^{i}-1\right)+1}, \ldots, x_{k^{i+1}}\right)\right) .
\end{aligned}
$$

Since $t$ is idempotent, it is easy to see that $t_{i}$ is a weak near-unanimity term for all $i$. Moreover, $t_{i}(y, x, \ldots, x)=\tau_{x}^{i}(y)$ (the $i$ th iterate of $\tau_{x}$ applied to $y$ ) where $\tau_{x}$ is the unary operation on $A$ defined by $\tau_{x}(y)=t(y, x, \ldots, x)$. The $n$ !-fold iteration of a unary operation on an $n$-element set yields an idempotent operation; that is, $\tau_{x}^{2 n !}(y)=\tau_{x}^{n !}(y)$ for all $x, y \in A$. This means that $t_{n !}$ is a special weak nearunanimity term for $\mathbf{A}$.

Lemma 4.8. If an algebra has a $k+1$-ary special weak near-unanimity term, then it has an $m k+1$-ary special weak near-unanimity term for every integer $m \geq 1$. 
Proof. Let $T$ be a special weak $k$-nu for $\mathbf{A}$. We define by induction a sequence $t_{1}, t_{2}, \ldots$ of terms of arities $k+1,2 k+1, \ldots$, respectively. Put $t_{1}=t$. If $t_{m}$ is defined, then define

$$
t_{m+1}\left(x_{0}, \ldots, x_{(m+1) k}\right)=t_{m}\left(t\left(x_{0}, \ldots, x_{k}\right), x_{k+1}, \ldots, x_{(m+1) k}\right) .
$$

Clearly, by an inductive argument, $t_{i}$ is a special weak-nu term for each $i \geq 1$.

Lemma 4.9. Let $\mathbf{A}$ be a finite algebra of size $n$, with a special weak near-unanimity term $t$ of arity $k+1$, and let $m \geq n k$. Then $\mathbf{A}$ satisfies $T S(m k+1)$.

Proof. Let $\mathbf{B} \leq \mathbf{A}^{m k+1}$ be a totally symmetric subalgebra.

Claim 4.10. Suppose that $a b^{k} \bar{x} \in B$ for some $a, b \in A$ and $\bar{x} \in A^{(m-1) k}$. Then $c^{k+1} \bar{x} \in B$ where $c=t(a, b, \ldots, b)$.

For $0 \leq i \leq k$ let $f_{i}=b^{i} a b^{k-i} \bar{x}$. Since $B$ is totally symmetric, $f_{i} \in B$ for all $i$. Then $c^{k+1} \bar{x}=t\left(f_{0}, \ldots, f_{k}\right) \in B$.

Claim 4.11. Suppose that $a^{i} b^{j k} \bar{x} \in B$ for some integers $1 \leq i \leq j$ where $a, b \in A$. Then $c^{i+j k} \bar{x} \in B$ where $c=t(a, b, \ldots, b)$.

By applying the previous claim to the coordinates that contain $a b^{k}$ we get that $c^{k+1} a^{i-1} b^{(j-1) k} \bar{x} \in B$. By repeated applications we obtain that $c^{i+i k} b^{(j-i) k} \bar{x} \in B$. Note, that $t(c, b \ldots, b)=c$ because $t$ is special. Therefore, by applying the previous claim to the coordinates that contain $c b^{k}$, we get that $c^{i+(i+1) k} b^{(j-i-1) k} \bar{x} \in B$. By repeated applications we finally obtain that $c^{i+j k} \bar{x} \in B$.

ClaIm 4.12. Suppose that $a^{k^{2}} b_{0} \ldots b_{k} \bar{x} \in B$ for some elements $a, b_{0}, \ldots b_{k}$ in $A$ and tuple $\bar{x}$. Then $c_{0}^{k} \ldots c_{k}^{k} d \bar{x} \in B$ for some elements $c_{0}, \ldots, c_{k}, d$ in $A$.

For $1 \leq i \leq k$ and $0 \leq j \leq k$ put $\bar{y}_{i, j}=a^{i-1} b_{j} a^{k-i} \in A^{k}$. For $0 \leq i \leq k$ define $f_{i}=\bar{y}_{i, 0} \ldots \bar{y}_{i, i-1} a^{k} \bar{y}_{i+1, i+1} \ldots \bar{y}_{i+1, k} b_{i} \bar{x}$, which is best described by the following matrix:

$$
\left[\begin{array}{c}
f_{0} \\
f_{1} \\
f_{2} \\
\vdots \\
f_{k-1} \\
f_{k}
\end{array}\right]=\left[\begin{array}{ccccccccccccccc}
a & a & \cdots & a & b_{1} & a & \cdots & a & \cdots & b_{k} & a & \cdots & a & b_{0} & \bar{x} \\
b_{0} & a & \cdots & a & a & a & \cdots & a & \cdots & a & b_{k} & \cdots & a & b_{1} & \bar{x} \\
a & b_{0} & \cdots & a & a & b_{1} & \cdots & a & \cdots & a & a & \cdots & a & b_{2} & \bar{x} \\
& & \vdots & & & & \vdots & & \vdots & & & \vdots & & \vdots \\
a & a & \cdots & a & a & a & \cdots & a & \cdots & a & a & \cdots & a & b_{k-1} & \bar{x} \\
a & a & \cdots & b_{0} & a & a & \cdots & b_{1} & \cdots & a & a & \cdots & a & b_{k} & \bar{x}
\end{array}\right]
$$

Clearly, $f_{0}, \ldots, f_{k} \in B$, because each is obtained from $a^{k^{2}} b_{0} \ldots b_{k} \bar{x}$ by permuting its coordinates. By calculating $f=t\left(f_{0}, \ldots, f_{k}\right) \in B$ we see that $f=c_{0}^{k} \ldots c_{k}^{k} d \bar{x}$, where $c_{i}=t\left(b_{i}, a, \ldots, a\right)$ and $d=t\left(b_{0}, \ldots, b_{k}\right)$.

CLAIM 4.13. There exist elements $a_{1}, a_{2} \ldots, a_{m}, b \in A$ so that $a_{1}^{k} a_{2}^{k} \ldots a_{m}^{k} b \in B$.

Let $j \leq m$ be the largest integer for which there exist elements $a_{1}, \ldots, a_{j}$, $b_{0}, \ldots, b_{(m-j) k} \in A$ so that $a_{1}^{k} \ldots a_{j}^{k} b_{0} \ldots b_{(m-j) k} \in B$. To get a contradiction, assume that $j<m$. Clearly $m-j<n$, otherwise some element of $A$ appears at least $k$-many times in $b_{0}, \ldots, b_{n k}$ by the pigeon-hole principle, which contradicts the maximality of $j$. Consequently, $n(k-1) \leq m-n<j$. Applying the pigeon-hole principle again to the elements $a_{1}, \ldots, a_{j}$ we may assume that $a_{1}=a_{2}=\ldots a_{k}$. Therefore, 
$a_{1}^{k^{2}} b_{0} \ldots b_{k} a_{k+1}^{k} \ldots a_{j}^{k} b_{k+1} \ldots b_{(m-j) k} \in B$. Applying the previous claim to this tuple yields elements $c_{0}, \ldots, c_{k}, d \in A$ so that $c_{0}^{k} \ldots c_{k}^{k} d a_{k+1}^{k} \ldots a_{j}^{k} b_{k+1} \ldots b_{(m-j) k} \in B$. But this contradicts the maximality of $j$.

Claim 4.14. There exist elements $b, c \in A$ so that $c^{m k} b \in B$.

Let $j \leq m$ be the largest integer for which there exist elements $b, c \in A$ and $a_{j+1}, \ldots, a_{m} \in A$ so that $c^{j k} a_{j+1}^{k} \ldots a_{m}^{k} b \in B$. Clearly, $k \leq j$ by applying the pigeon-hole principle to the tuple $a_{1}^{k} \ldots a_{m}^{k} b$ whose existence is guaranteed by the previous claim. To get a contradiction, assume that $j<m$. Using Claim 4.11 for the tuple $a_{j+1}^{k} c^{j k} a_{j+2}^{k} \ldots a_{m}^{k} b$ we get that $d^{(j+1) k} a_{j+2}^{k} \ldots a_{m}^{k} b \in B$ for some $d \in A$, which is a contradiction.

Claim 4.15. There exists an element $a \in A$ so that $a^{m k+1} \in B$.

This follows immediately from the previous claim and Claim 4.11.

Theorem 4.16. Let $\mathbf{A}_{1}, \ldots, \mathbf{A}_{n}$ be finite algebras of the same signature such that for $1 \leq i \leq n, \mathbf{A}_{i}=\mathrm{WNU}\left(k_{i}\right)$ for some $k_{i}>1$. There is an integer $k>0$ and a term $t(\bar{x})$ of $k+1$ variables in the signature of these algebras so that for all $1 \leq i \leq n, t$ is a special weak near-unanimity operation for $\mathbf{A}_{i}$ and for all $m \geq 1$, $\mathbf{A}_{i} \models \operatorname{TS}(m k+1)$.

Proof. It follows easily from Lemmas 4.7, 4.8 and 4.9 that there is an integer $N>1$ such that every algebra $\mathbf{A}_{i}$ satisfies $\operatorname{TS}(N)$. Let $\mathbf{F}$ be the free algebra on two generators $x, y$ in the variety generated by $\mathbf{A}_{1}, \ldots, \mathbf{A}_{n}$. By Lemma 4.4, $\mathbf{F} \models \operatorname{TS}(N)$, and by Lemma 4.5, (iii), $\mathbf{F}=\mathrm{WNU}(N)$. Then by Lemma $4.7, \mathbf{F}$ has a special weaknu term of $K+1$ variables for a certain $K>0$. This term is also a special weak-nu term for each $\mathbf{A}_{i}$. Let $M$ be the cardinality of the largest algebra among the $\mathbf{A}_{i}$. Then by Lemma 4.9 , when $m \geq M K$ then every algebra $\mathbf{A}_{i}$ satisfies $\operatorname{TS}(m K+1)$. We take $k=M K^{2}$. Thus for $m \geq 1$, each $\mathbf{A}_{i}$ satisfies $\operatorname{TS}(m k+1)$. Moreover, our proof of Lemma 4.8, starting with the special weak-nu term of $K+1$ variables for $\mathbf{F}$, yields a term $t(\bar{x})$ of $k+1$ variables that is a special weak-nu for each of the algebras $\mathbf{A}_{i}$.

There is another set of hypotheses that forces satisfaction of $\operatorname{TS}(k)$.

Theorem 4.17. Suppose that $\mathbf{A}$ is a finite algebra. Let $m \geq 3$ and $k \geq(m-1)|\mathbf{A}|$. If $\mathbf{A}$ satisfies $\mathrm{ST}(n)$ for all $m \leq n \leq k$ then $\mathbf{A}$ satisfies $\operatorname{TS}(k)$.

Proof. Let $T$ be a non-empty totally symmetric subuniverse of $\mathbf{A}^{k}$. Choose $\bar{x} \in T$. Since $k \geq(m-1)|\mathbf{A}|$, and by total symmetry, we can assume that

$$
\bar{x}=c^{m-1} x_{m} \cdots x_{k}=c^{m-1} \bar{x} .
$$

Now let $n$ be maximal, $m-1 \leq n \leq k$, so that $T$ contains an element of the form $u^{n} \bar{z}, u \in A$. Let $a^{n} \bar{q} \in T, a \in D(T)$. We can assume that $n<k$, else the proof is finished. Write $\bar{q}=b \bar{p}, b \in D(T)$. Put

$$
S=\left\{\bar{x} \in A^{n+1}: \bar{x} \bar{p} \in T\right\} .
$$

Obviously, $S$ is a totally symmetric subuniverse of $\mathbf{A}^{n+1}$ (since the operations are idempotent and $T$ is totally symmetric). Moreover $S$ contains the vector $a^{n} b$. Thus $S$ contains a $\star$-subalgebra of $\mathbf{A}^{n+1}$. Since $n \geq m-1$, then $m \leq n+1 \leq k$. Then since $\operatorname{ST}(n+1)$ is satisfied by $\mathbf{A}$, we conclude that there is $e \in A$ with $e^{n+1} \in S$. This means that $e^{n+1} \bar{p} \in T$. But this contradicts the assumed maximality of $n$. 


\section{Minimal Star subalgebras}

If we are trying to prove that a given finite algebra satisfies $\mathrm{ST}(k)$, then we will be looking at a $\star$-subalgebra $\mathbf{B} \leq \mathbf{A}^{k}$, and attempting to show that $\mathbf{B}$ contains a diagonal element. Among all the $\star$-subalgebras included in $\mathbf{B}$, there will be at least one that properly includes no other $\star$-subalgebra of $\mathbf{A}^{k}$. Every $\star$-subalgebra of $\mathbf{A}^{k}$ contains a diagonal vector iff every one of these minimal $\star$-subalgebras of $\mathbf{A}^{k}$ is a singleton, consisting of a diagonal vector. Thus it is natural to focus our attention on minimal $\star$-subalgebras of $\mathbf{A}^{k}$. These algebras, in general, have some very interesting properties.

Definition 5.1. Let $\mathbf{T}$ be a minimal $\star$-subalgebra of $\mathbf{F}^{k}$ where $\mathbf{F}$ is a finite algebra and $k \geq 3$. Thus $D(T)$ (defined in Definition 4.1 as the projection of $T$ onto the first coordinate, where $T$ is the universe of $\mathbf{T}$ ), is a two-generated subuniverse of $\mathbf{F}$. For $a, b \in D(T)$ we write $b \prec a$ iff $b a^{k-1} \in T$. We say that $\langle a, b\rangle$ is a primitive pair for $\mathbf{T}$ when $b \prec a$. Notice that $b \prec a$ entails that $\{a, b\}$ generates $D(T)$.

We call an element $a \in D(T)$ generic with respect to $\mathbf{T}$ iff $b \prec a$ for some $b \in D(T)$. We call an element $b \in D(T)$ co-generic with respect to $\mathbf{T}$ iff $b \prec a$ for some $a \in D(T)$. A two-generator of $D(T)$ is an element $u$ such that for some $v \in D(T),\{u, v\}$ generates $D(T)$. Thus every element of $D(T)$ that is generic or co-generic with respect to $\mathbf{T}$ is a two-generator of $D(T)$.

Lemma 5.2. Let $\mathbf{T}$ be a minimal $\star$-subalgebra of $\mathbf{F}^{k}$ where $\mathbf{F}$ is a finite algebra and $k \geq 3$. For $a \in D(T)$ the following are equivalent.

(i) a is a generic element with respect to $\mathbf{T}$.

(ii) There is some $\bar{x}=x_{1} x_{2} \cdots x_{k} \in T$ such that $x_{1}$ is co-generic with respect to $\mathbf{T}$ and $x_{2}=a$.

(iii) For all $\bar{w} \in T$ there is $\bar{y} \in T$ with $w_{i}=y_{i}$ for $3 \leq i \leq k$ and $w_{1}=a$. Also, the following are equivalent.

(a) There are $u, v, w \in D(T)$ with $u \prec v \prec w$.

(b) For all $v \in D(T)$ there are $u, w \in D(T)$ with $u \prec v \prec w$; i.e., every element of $D(T)$ is both generic and co-generic with respect to $\mathbf{T}$.

(c) For all $\bar{x} \in D(T)^{k}$ there is $\bar{y} \in T$ with $x_{i}=y_{i}$ for $1 \leq i \leq k-1$.

Proof. First, we tackle (iii) $\Rightarrow$ (i). Take any $\bar{x}$ in $\mathbf{T}$. Choose $\bar{y}^{1} \in \mathbf{T}$ with $y_{1}^{1}=a$. Exchanging the first and third coordinates and applying (iii), we find some axa $\cdots$ in $T$. If $3<k$, then exchange the first and fourth co-ordinates in this vector and apply (iii) to get some ayaa $\cdots$ in $T$. This procedure can be continued, and eventually produces $a b a^{k-2} \in T$ for some $b \in D(T)$. This is equivalent to $b \prec a$. Thus $a$ is generic with respect to $T$.

The implication (i) $\Rightarrow$ (ii) is trivial, since $b \prec a$ means that $b a \cdots a \in T$.

For (ii) $\Rightarrow$ (iii), suppose that $c \prec d, \bar{x} \in \mathbf{T}$ and $x_{1}=c, x_{2}=a$. Since $\mathbf{T}$ is a minimal $\star$-subalgebra, the vectors $\varepsilon_{1}, \ldots, \varepsilon_{k}$ defined by $\varepsilon_{i}(i)=c$ and $\varepsilon_{i}(j)=d$ when $i \neq j$ constitute a generating set for $\mathbf{T}$. Thus we can write $\bar{x}=t\left(\varepsilon_{1}, \ldots, \varepsilon_{k}\right)$ in $\mathbf{T}$ for some term $t$. Then

$$
t\left(\varepsilon_{3}, \varepsilon_{1}, \varepsilon_{2}, \ldots, \varepsilon_{2}\right)=\bar{y}
$$

is a vector in $T$ of the form $a p c d^{k-3}$ for some $p \in D(T)$. Likewise

$$
t\left(\varepsilon_{2}, \varepsilon_{1}, \varepsilon_{2}, \ldots, \varepsilon_{2}\right)=\bar{z}
$$


is a vector of the form $a q d^{k-2}$ where $q \in D(T)$. We write $\tau_{1}=\bar{z}=a q d^{k-2}$, and for $3 \leq i \leq k$ we put $\tau_{i}=a p d^{i-3} c d^{k-i}$. All these vectors can be obtained by permuting the coordinates in $\bar{y}$ or $\bar{z}$ and so, they belong to $T$.

Now suppose that $\bar{w}$ is any vector in $T$. Write $\bar{w}=s\left(\varepsilon_{1}, \ldots, \varepsilon_{k}\right)$ for a term $s$, just as we wrote $\bar{x}$ above. Consider

$$
\bar{w}^{\prime}=s\left(\tau_{1}, \tau_{1}, \tau_{3}, \ldots, \tau_{k}\right) .
$$

It is easily verified that $w(i)=w^{\prime}(i)$ for $3 \leq i \leq k$ and that $w^{\prime}(1)=a$. This concludes our proof that (ii) $\Rightarrow$ (iii).

For proving the equivalence of (a), (b), (c), we first note that (c) $\Rightarrow$ (a) is easy: Taking any $c \in D(T)$, and taking $\bar{x}=c^{k-1}$, there is $b c^{k-1} \in T$ for some $b$, i.e., $b \prec c$. By the same token, there is some $a \in D(T)$ with $a \prec b$.

For $(\mathrm{a}) \Rightarrow(\mathrm{b})$, choose three elements of $D(T)$ satisfying $a \prec b \prec c$ and let $u$ be any element of $D(T)$. Find $\bar{x} \in T$ with $x_{1}=u$. Write first $\bar{x}=s\left(\tau_{1}, \ldots, \tau_{k}\right)$ where $\tau_{i}(i)=a$ and $\tau_{i}(j)=b$ when $i \neq j$. Consider

$$
\bar{y}=s\left(\tau_{1}, \gamma, \ldots, \gamma\right)
$$

where $\gamma=b c \cdots c \in T$. We have $\bar{y}=u v \cdots v$ where $v=s(b, c, \ldots, c)$, i.e., $u \prec v$. Now write $\bar{x}=t\left(\gamma_{1}, \ldots, \gamma_{k}\right)$ where $\gamma_{i}(i)=b$ and $\gamma_{i}(j)=c$ when $i \neq j$. Consider

$$
\bar{z}=t\left(\tau_{k}, \gamma_{k}, \ldots, \gamma_{k}\right) .
$$

We have $\bar{z}=u^{k-1} w$ where $w=t(a, b, \ldots, b)$. Thus also $w u^{k-1} \in T$ and $w \prec u$. We have shown, given $u \in D(T)$, that there exist $v, w \in D(T)$ with $w \prec u \prec v$. The derivation of $(\mathrm{a}) \Rightarrow(\mathrm{b})$ is finished.

To prove that $(\mathrm{b}) \Rightarrow(\mathrm{c})$, we use the implication (i) $\Rightarrow$ (iii). Assume that (b) holds. Then every element $a \in D(T)$ is generic with respect to $T$. Thus every element $a \in D(T)$ satisfies (iii). Now, given $\bar{x} \in D(T)^{k}$, we can choose a vector $x_{1} \bar{y} \in T$. Exchanging the first and third coordinates in this vector and applying (iii) (with $a=x_{2}$ ) we get a vector $x_{2} p x_{1} \bar{z} \in T$, and then after a permutation of coordinates, we have a vector $p q x_{1} x_{2} \bar{w} \in T$. We apply (iii) to this vector with $a=x_{3}$, and obtain a vector $x_{3} r x_{1} x_{2} \bar{w} \in T$. Clearly we can continue in this fashion to eventually obtain $x_{k-1} s x_{1} x_{2} \cdots x_{k-2} \in T$ for some $s \in D(T)$. After a permutation of coordinates, we get $x_{1} x_{2} \cdots x_{k-1} s \in T$, as required.

Definition 5.3. A minimal $\star$-subalgebra of $\mathbf{F}^{k}$ satisfying the equivalent conditions (a)-(b)-(c) of Lemma 5.2 will be called $k-1$-complete.

Lemma 5.4. Let $\mathbf{F}$ be a finite algebra and $k \geq 3$. If $\mathbf{F}$ satisfies $\operatorname{WNU}(k-1)$, $\mathrm{WNU}(k)$ or $\mathrm{WNU}(k+1)$, then every minimal $\star$-subalgebra of $\mathbf{F}^{k}$ is $k$-1-complete.

Proof. Let $\mathbf{T}$ be any minimal $\star$-subalgebra of $\mathbf{F}^{k}$. If $\mathbf{F}$ satisfies WNU $(k)$ then clearly, $\mathbf{T}$ has a diagonal vector $a^{k}$, implying that $T=\left\{a^{k}\right\}$ and $D(T)=\{a\}$. Clearly $\mathbf{T}$ is $k-1$-complete in this case.

Assume that $\mathbf{F}$ satisfies $\mathrm{WNU}(k-1)$. Let $m\left(x_{1}, \ldots, x_{k-1}\right)$ be a $k-1$-WNU term for $\mathbf{F}$. Choose any $a \prec b$ in $D(T)$. Consider the vector

$$
\bar{x}=m\left(a b^{k-1}, b a b^{k-2}, b b a b^{k-3}, \ldots, b^{k-2} a b\right) .
$$

This vector in $\mathbf{T}$ has the form $c^{k-1} b$. So we have $a \prec b \prec c$. Now by Lemma 5.2 and Definition 5.3 it follows that $\mathbf{T}$ is $k-1$-complete. 
Now assume that $\mathbf{F}$ satisfies $\mathrm{WNU}(k+1)$. Let $w\left(x_{1}, \ldots, x_{k+1}\right)$ be a $k+1-\mathrm{WNU}$ term for $\mathbf{F}$. Choose $u, v \in D(T)$ such that $u \prec v$. Put $p=w(u, u, v \ldots, v)$, $q=w(v, \ldots, v, u, u), v^{\prime}=w(u, v, \ldots, v)$. We find that $p v^{\prime} \cdots v^{\prime} \in T$ by giving

$$
u v^{k-1}, u v^{k-1}, v u v^{k-2}, \ldots, v^{k-1} u
$$

as arguments to $w$ in $\mathbf{T}$. Thus $p \prec v^{\prime}$. To complete the proof of this theorem, it will suffice, by Lemma 5.2, to show that $p$ is generic with respect to $\mathbf{T}$.

Analogously to the above, we have that $q \prec v^{\prime}$. Also, we have that

$$
p q v^{\prime} \cdots v^{\prime} v \in T,
$$

as can be demonstrated by giving $w$ these arguments in $\mathbf{T}$ :

$$
\begin{gathered}
u v^{k-1}, u v^{k-1}, v v u v^{k-3}, v v v u v^{k-4}, \ldots, \\
v^{k-2} u v, v u v^{k-2}, v u v^{k-2} .
\end{gathered}
$$

Now since $p q \cdots \in T$ and $p, q$ are both co-generic then, by Lemma 5.2 (equivalence of (i)-(ii)-(iii)), $p, q$ are also both generic. Thus $\mathbf{T}$ is $k-1$-complete by Lemma 5.2 .

Lemma 5.5. Let $\mathbf{F}$ be a finite algebra and $k \geq 3$. Let $\mathbf{T}$ be a minimal $\star$-subalgebra of $\mathbf{F}^{k}$. Suppose that there is $c_{1} c_{2} \cdots c_{k} \in T$ where every $c_{i}$ is generic with respect to $\mathbf{T}$. Then $T$ is $k-1$-complete.

Proof. We claim that if $y \in D(T)$ then $y c_{2} c_{3} \cdots c_{k-1} u \in T$ for some generic element $u$. Indeed, let $c_{1}^{k-1} b \in T$. Then we can write $y=s\left(c_{1}, b\right)$ for some term $s$. Now there is $b c_{2} c_{3} \cdots c_{k-1} c \in T$ for some $c$ since $c_{2}, c_{3}, \ldots$ are generic (using $k-2$ applications of the equivalence (i) $\leftrightarrow$ (iii) in Lemma 5.2). Here $c$ is generic by the equivalence (i) $\leftrightarrow$ (ii) in Lemma 5.2 (since $b$ is co-generic). Now in $\mathbf{T}$ we have the equation

$$
s\left(c_{1} c_{2} \cdots c_{k}, b c_{2} \cdots c_{k-1} c\right)=y c_{2} c_{3} \cdots c_{k-1} s\left(c_{k}, c\right)
$$

and $s\left(c_{k}, c\right)$ is generic, since $c_{k}, c$ are generic (and the set of generic elements of $D(T)$ is obviously a subalgebra).

The claim obviously generalizes: if $y \in D(T)$ and $d_{1} \cdots d_{k} \in T$ where all $d_{i}$ are generic, then $y d_{2} \cdots d_{k-1} u \in T$ for some generic $u$. Applying this claim to $c_{2} c_{1} c_{3} \cdots c_{k}$ and to $y=c_{1}$, we find that $c_{1} c_{1} c_{3} \cdots c_{k-1} u \in T$ for some generic element $u$. Then applying the claim to $c_{3} c_{1} c_{1} c_{4} \cdots c_{k-1} u$ and with $y=c_{1}$, we get that $c_{1} c_{1} c_{1} c_{4} \cdots c_{k-1} v \in T$ for some generic $v$. Obviously, we can continue to replace $c_{4}, c_{5}, \ldots$ by $c_{1}$ and we eventually will obtain that $c_{1}^{k-1} q \in T$ for some generic element $q$. Now $q$ is both generic and co-generic. Finally, it follows from the equivalence $(\mathrm{a}) \leftrightarrow(\mathrm{c})$ in Lemma 5.2 that $T$ is $k-1$-complete.

\section{Characteristic COngruence And two-Generators For a Minimal STAR SUBALGEBRA}

Definition 6.1. Let $\mathbf{A}$ be an algebra and $k \geq 2$, and let $\mathbf{T}$ be a non-empty totally symmetric subalgebra of $\mathbf{A}^{k}$. For $\bar{x} \in A^{k-1}$, put $\operatorname{pr}_{\bar{x}}(T)=\{u \in a: u \bar{x} \in T\}$. Define $\rho_{T}$ to be the set of all pairs $(u, v) \in A^{2}$ such that for some $\bar{x} \in A^{k-1}$, $\{u, v\} \subseteq \operatorname{pr}_{\bar{x}}(T)$. For $x \in A$, and $n \geq 1$ we put $\rho_{T}^{n}(x)=\left\{y \in A:(x, y) \in \rho_{T}^{n}\right\}$ where $\rho_{t}^{n}$ is the $n$-fold relation composition of $\rho_{T}$ with itself, so that

$$
\rho_{T}^{1}=\rho_{T}, \rho_{T}^{2}=\left\{(u, v) \in A^{2}:(u, a),(a, v) \in \rho_{T} \text { for some } a \in D(T)\right\}, \text { etc. }
$$


Now, $\rho_{T}^{n}$ is a reflexive and symmetric subuniverse of $D(T)^{2}$, and $\rho_{T}^{n}(x)$ is a subuniverse of $D(T)$ containing $x$, whenever $x \in D(T)$. Considering the algebra $\mathbf{D}(T)$ with universe $D(T)$, let $\theta_{T}$ denote the congruence on $\mathbf{D}(T)$ generated by $\rho_{T}$. We call $\theta_{T}$ the characteristic congruence of $T$. Note that if $\mathbf{A}$ is finite, then there is a positive integer $n$ such that $\theta_{T}=\rho_{T}^{n}$.

Lemma 6.2. Let $\mathbf{A}$ be a finite algebra, $k \geq 2$, and $\mathbf{T}$ be a minimal $\star$-subalgebra of $\mathbf{A}^{k}$. If $|T|>1$ then for every $\bar{x} \in D(T)^{k-1}, p r_{\bar{x}}(T) \neq D(T)$.

Proof. Put $W=\left\{\bar{x} \in D(T)^{k-1}: \operatorname{pr}_{\bar{x}}(T)=D(T)\right\}$. To work toward a contradiction, suppose that $W \neq \emptyset$. Note that $W$ is a symmetric subuniverse of $D(T)^{k-1}$.

Choose $n$ maximal, $1 \leq n \leq k-1$, such that there is $c^{n} \bar{x} \in W$ for some $\bar{x} \in W^{k-n-1}$. Since $|T|>1$, then $n<k-1$ (else $c^{k-1} \in W$, implying $c^{k} \in T$, giving that $\left\{c^{k}\right\}$ is a $\star$-subalgebra of $\mathbf{A}^{k}$ contained in $T$, contradicting minimality of $T)$. Note that since $c^{k-1} \notin W$, then $k \geq 3$. Now choose any $b \in D(T)$ that is co-generic with respect to $\mathbf{T}$. We have that $b c^{n} \bar{x} \in T$. By Lemma 5.2, $c$ is generic with respect to $T$, so with the right choice of $b$, we can assume that $b c^{k-1} \in T$, as well as $b c^{n} \bar{x} \in T$ and $c c^{n} \bar{x} \in T$ ( $\operatorname{since} c^{n} \bar{x} \in W$ ).

Now we claim that for any $\bar{s} \in T$ we have also $s_{1} s_{2} \cdots s_{n+1} \bar{x} \in T$. The claim obviously will imply that for any $\bar{t} \in W$, we have also $t_{1} t_{2} \cdots t_{n} \bar{x} \in W$. To prove the claim, letting $\bar{s} \in T$, we choose a term $\lambda$ such that $\lambda\left(f_{1}, \ldots, f_{k}\right)=\bar{s}$ where $f_{i}$ is the vector with $b$ at the $i$ place and $c$ everywhere else. (Here we use the minimality of $T$.) Now in the equation

$$
\bar{s}=\lambda\left(f_{1}, \ldots, f_{k}\right),
$$

replace $f_{n+2}, \ldots, f_{k}$ by the vector $c^{n+1} \bar{x}$, which belongs to $T$, and for $1 \leq i \leq n+1$, replace $f_{i}$ by the vector obtained from $c^{n+1} \bar{x}$ by replacing $c$ at place $i$ by $b$. These vectors also belong to $T$. This yields a new equation

$$
s_{1} s_{2} \cdots s_{n+1} \bar{x}=\lambda\left(g_{1}, \ldots, g_{k}\right) \in T .
$$

The claim is proved.

Now recall from above that we have $n<k-1$. Thus write $\bar{x}=d \bar{y}$ for some $d \in D(T)$. So we have $c^{n} d \bar{y} \in W$, or equivalently, $c^{n-1} d c \bar{y} \in W$. By the claim, we get that $c^{n-1} d d \bar{y} \in W$ (replacing $c \bar{y}$ by $\bar{x}=d \bar{y}$ ). Then again, $c^{n-2} d d c \bar{y} \in W$; and this yields in the same fashion, $c^{n-2} d d d \bar{y} \in W$. Obviously, an inductive argument now gives that $d^{n+1} \bar{y} \in W$. But this contradicts our choice of $n$ as maximal. The contradiction finishes our proof that $\operatorname{pr}_{\bar{x}}(T)=D(T)$ is impossible.

Recall that we defined an element $a$ of an algebra $\mathbf{B}$ to be a two-generator of $\mathbf{B}$ just in case for some $b \in B$ we have that $\{a, b\}$ generates $\mathbf{B}$.

Lemma 6.3. Let $\mathbf{A}$ be a finite algebra and $k \geq 2$, and let $\mathbf{T}$ be a minimal $\star-$ subalgebra of $\mathbf{A}^{k}$. If $|T|>1$ then for every $x \in \bar{D}(T)$, if $\rho_{T}(x)=D(T)$ then $x$ is not a two-generator of $D(T)$.

Proof. Suppose this fails. Then we can choose $\{a, b\} \subseteq D(T)$ so that $\{a, b\}$ generates $D(T)$ and $\rho_{T}(a)=D(T)$. Thus $(a, b) \in \rho_{T}$. There is $\bar{x} \in D(T)^{k-1}$ with $\{a, b\} \subseteq \operatorname{pr}_{\bar{x}}(T)$. Since $\operatorname{pr}_{\bar{x}}(T)$ is a subalgebra of $D(T)$, then $\operatorname{pr}_{\bar{x}}(T)=D(T)$. This contradicts Lemma 6.2. 
Lemma 6.4. Let $\mathbf{A}$ be a finite algebra and $k \geq 2$, and let $\mathbf{T}$ be a minimal *subalgebra of $\mathbf{A}^{k}$. Suppose that $\{a, x, y\} \subseteq D(T)$ and a is generic with respect to $T$. If $a^{k-2} x y \in T$ then $x$ is a two-generator of $D(T)$ iff $y$ is a two-generator of $D(T)$.

Proof. Let $a^{k-2} x y \in T$ and suppose that $\{x, z\}$ generates $D(T)$. We have to show that $y$ is a two-generator. Since $a$ is generic, there is $a^{k-2} z w \in T$ for some $w$. Write $S=\operatorname{Sg}(\{y, w\})$. We show that $S=D(T)$, demonstrating that $y$ is a two-generator. Choose a term $t$ so that $t(x, z)=a$. Applying $t$ in $\mathbf{T}$ to $a^{k-2} x y, a^{k-2} z w$, we obtain that $a^{k-1} t(y, w) \in T$. Write $g=t(y, w)$. Thus $\langle a, g\rangle$ is a primitive pair for $\mathbf{T}$. Choose a term $s$ so that $s(x, z)=g$. Then applying $s$ in $\mathbf{T}$ to $a^{k-2} x y$ and $a^{k-2} z w$, we get that $a^{k-2} g s(y, w) \in T$. Write $u=s(y, w)$, so that $u$ is a generic element, since $g$ is co-generic and $a^{k-2} g u \in T$ (by an application of Lemma 5.2). Now we have $\{u, g\} \subseteq S, a^{k-1} g \in T, a^{k-2} g u \in T$. Choose $h$ so that $u^{k-1} h \in T$.

We claim that for any $\bar{x} \in T$ there is $s \in S$ with $x_{1} x_{2} \cdots x_{k-1} s \in T$. If this is true, then there must be $s \in S$ with $u^{k-1} s \in T$. Then $\langle u, s\rangle$ is a primitive pair with respect to $T$, and $S$ contains $\{u, s\}$, implying that $S=D(T)$, which is what we wished to prove. To prove the claim, let $\bar{x} \in T$ and write

$$
\bar{x}=\sigma\left(\rho_{1}, \ldots, \rho_{k}\right),
$$

where the vector $\rho_{i}$ has $a$ at all places but the $i$ th, and $g$ at the $i$ th place. Define $\tau_{i}$ $(1 \leq i<k)$ to agree with $\rho_{i}$ except at the $k$ th place, where $\tau_{i}(k)=u$. The vectors $\tau_{i}$ belong to $T$, since $a^{k-2} g u \in T$. Thus

$$
\left\{\tau_{1}, \tau_{2}, \ldots, \tau_{k-1}, \rho_{k}\right\} \subseteq T .
$$

Now

$$
\sigma\left(\tau_{1}, \tau_{2}, \ldots, \tau_{k-1}, \rho_{k}\right)=\bar{x}^{\prime}
$$

where $\bar{x}^{\prime}(i)=\bar{x}(i)$ for $1 \leq i<k$, and $\bar{x}^{\prime}(k)=\sigma(u, \ldots, u, g)=s \in S$. This proves the claim.

Definition 6.5. An algebra A satisfies TST $(k)$ iff every non-empty totally symmetric subalgebra of $\mathbf{A}^{k}$ contains a $\star$-subalgebra of $\mathbf{A}^{k}$.

Theorem 6.6. Assume that $\mathbf{T}$ is a minimal $\star$-subalgebra of $\mathbf{A}^{k}$ where $\mathbf{A}$ is a finite algebra and $k \geq 3$, and assume further that $|\mathbf{T}|>1$. Assume also that every proper subalgebra of A satisfies $\operatorname{TST}(k-1)$. Then $\theta_{T}$, the characteristic congruence for $\mathbf{T}$, is distinct from $D(T) \times D(T)$.

Proof. In search of a contradiction, we assume that $\theta_{T}=D(T) \times D(T)$. Thus there is a positive integer $n$ such that $\rho_{T}^{n}=D(T) \times D(T)$. Choose $n_{0}$ to be the least such positive integer.

We claim that $n_{0}=1$. To prove this, suppose that $n_{0}>1$. Choose a primitive pair $\langle a, b\rangle$ for $T$. We can also choose some $u \in D(T)$ so that $(a, u) \in \rho_{T}^{n_{0}-1}$ and $(u, b) \in \rho_{T}$. Our immediate aim is to prove that $u$ must be a two-generator of $D(T)$.

By definition, we can choose some $\bar{x} \in A^{k-1}$ so that $u \bar{x}, b \bar{x} \in T$. Since $b \bar{x} \in T$, then by Lemma $5.2, x_{i}$ is generic for $1 \leq i \leq k-1$. Rewriting, we have $\bar{x} u \in T$. Write $G$ for the set of all elements of $D(T)$ generic with respect to $T$. Then $G$ is a subalgebra of $D(T)$ and $\bar{x} \in G^{k-1}$. Define

$$
S=\left\{\bar{y} \in G^{k-1}: \bar{y} u \in T\right\} .
$$


Since $\bar{x} u \in T$, then $S$ is a non-empty subalgebra of $G^{k-1} . S$ is clearly totally symmetric. Now if $G=A$ then $u \in G$, implying that $u$ is a two-generator, as we hope. If $G \neq A$, then this algebra satisfies $\operatorname{TST}(k-1)$. We have then that $S$ contains a vector $a^{k-2} c$ for some $\{a, c\} \subseteq G$.

At this point, we have that $a^{k-2} c u \in T$ and $a$, and $c$ are both generic with respect to $T$, implying that $c$ is a two-generator of $D(T)$. Now Lemma 6.4 implies that $u$ is a two-generator.

Thus, indeed, $u$ must be a two-generator of $D(T)$. Choose $v$ so that $\{u, v\} \subseteq$ $D(T)$ and $\{u, v\}$ generates $D(T)$. Recall that $(u, a) \in \rho_{T}^{n_{0}-1}$ and $(u, b) \in \rho_{T} \subseteq$ $\rho_{T}^{n_{0}-1}$. Thus $\{a, b\} \subseteq \rho_{T}^{n_{0}-1}(u)$, implying that the algebra $\rho_{T}^{n_{0}-1}(u)$ is identical with $D(T)$. Consequently, $(u, v) \in \rho_{T}^{n_{0}-1}$. But since our algebras are idempotent, and $\{u, v\}$ generates $D(T)$, then the set of pairs

$$
\{(u, u),(v, v),(u, v),(v, u)\}
$$

generates $D(T) \times D(T)$. Thus it must be that $\rho_{T}^{n_{0}-1}=D(T) \times D(T)$. This contradicts the minimality of $n_{0}$. The contradiction establishes that, in fact, $\rho_{T}=$ $D(T) \times D(T)$.

Now we have $(a, b) \in \rho_{T}$, implying that $\rho_{T}(a)=D(T)$. Since $a$ is a two-generator of $D(T)$, this is a contradiction of Lemma 6.3 .

Theorem 6.7. Assume that $\mathbf{T}$ is a minimal $\star$-subalgebra of $\mathbf{A}^{k}$ where $\mathbf{A}$ is a finite simple algebra and $k \geq 3$. Assume also that $D(T)=A$ and that every proper subalgebra of $\mathbf{A}$ satisfies $\operatorname{TST}(k-1)$. Then $\mathbf{A}$ is an Abelian algebra.

Proof. Under the assumptions in this theorem, Theorem 6.6 implies that $\theta_{T}$ is the identity relation on $A$. Thus $\rho_{T}$ is the identity relation on $A$ as well. We now consider three congruences on the algebra $\mathbf{T}$. They are the kernels of the projections of $\mathbf{T}$ onto $\mathbf{A}$ at the first coordinate, and at the second coordinate, and the kernel of the projection of $\mathbf{T}$ into $\mathbf{A}^{k-2}$ over all remaining coordinates. Label these congruences $\sigma_{1}, \sigma_{2}$ and $\sigma_{1,2}^{\prime}$. Thus for $\bar{x}, \bar{y} \in T$ we have

$$
\begin{gathered}
(\bar{x}, \bar{y}) \in \sigma_{1} \leftrightarrow x_{1}=y_{1} \\
(\bar{x}, \bar{y}) \in \sigma_{2} \leftrightarrow x_{2}=y_{2} \\
(\bar{x}, \bar{y}) \in \sigma_{1,2}^{\prime} \leftrightarrow x_{i}=y_{i} \text { for all } 3 \leq i \leq k
\end{gathered}
$$

The remainder of this argument relies on the centralizer theory and theory of the solvability congruence on the congruence lattice of a finite algebra, as detailed in D. Hobby, R. McKenzie [6], Chapters 3 and 7.

Notice that $\rho_{T}=0_{A}$ (the identity relation on $A$ ) is equivalent to $\sigma_{2} \wedge \sigma_{1,2}^{\prime}=0_{T}$ and since $T$ is totally symmetric, also equivalent to $\sigma_{1} \wedge \sigma_{1,2}^{\prime}=0_{T}$. Let us put $\sigma=\sigma_{1} \vee \sigma_{2}$, and $\alpha=\sigma \wedge \sigma_{1,2}^{\prime}$. The fact that $\sigma_{i}$ is disjoint from $\sigma_{1,2}^{\prime}$ (for $i \in\{1,2\}$ ) implies that $\sigma_{i}$ centralizes $\sigma_{1,2}^{\prime}$. This implies that $\sigma$ centralizes $\sigma_{1,2}^{\prime}$, and thus $\alpha$ centralizes $\alpha$. The congruence $\alpha$ on $\mathbf{T}$ is Abelian. Thus $\sigma_{1}$ and $\sigma_{1} \vee \alpha$ are solvably equivalent.

Choose a primitive pair $\langle a, b\rangle$ for $T$. It is easy to see that $b a^{k-1}$ and $a b a^{k-2}$ are congruent modulo $\alpha$. Thus $a$ and $b$ are congruent modulo the image of $\sigma_{1} \vee \alpha$ under the projection map $\mathbf{D}(T) \rightarrow \mathbf{A}$ through the first coordinate. Thus this image is a non-identity congruence of $\mathbf{A}$; it can only be $A \times A$ since it contains the pair $(a, b)$, and $\mathbf{A}$ is simple. But since $\sigma_{1}$ and $\sigma_{1} \vee \alpha$ are solvably equivalent, this image is a 
solvable congruence of $\mathbf{A}$. This means that $A \times A$ is a solvable congruence. Since $\mathbf{A}$ is simple, we are forced to the conclusion that $\mathbf{A}$ is Abelian.

\section{Proof of Theorem 1.1}

Of the three conditions whose equivalence Theorem 1.1 asserts, (3) trivially implies (2), and we saw in Section 2 that (2) implies (1). The five Lemmas in Section 4 together with Theorem 4.16 show that (2) and (3) are equivalent. To complete a proof of the theorem, what remains is to show that in a locally finite variety that omits type $\mathbf{1}$, every finite algebra (or just the free algebra on two generators) satisfies $\operatorname{TS}(k)$ for some integer $k>1$.

So let $\mathcal{V}$ be a locally finite variety that omits type $\mathbf{1}$ and assume that $\mathcal{V}$ has a finite algebra $\mathbf{A}$ that does not satisfy $\operatorname{TS}(k)$ for any integer $k>1$. We shall derive a contradiction. We assume that the cardinality of $\mathbf{A}$ is as small as possible. This means that whenever $\mathbf{B} \in \mathcal{V}$ and $|\mathbf{B}|<|\mathbf{A}|$ then $\mathbf{B}$ satisfies $\operatorname{TS}(k)$ for some $k>1$. We choose a finite sequence of algebras $\mathbf{A}_{1}, \mathbf{A}_{2}, \ldots, \mathbf{A}_{n}$ so that $\mathbf{A}_{i} \in \mathcal{V}$ and $\left|\mathbf{A}_{i}\right|<|\mathbf{A}|$ for $1 \leq i \leq n$, and every algebra $\mathbf{B} \in \mathcal{V}$ with $|\mathbf{B}|<|\mathbf{A}|$ is isomorphic to some $\mathbf{A}_{i}$. With an application of Theorem 4.16, we choose an integer $k_{0}>1$ and a term $t_{0}(\bar{x})$ of $k_{0}+1$ variables in the signature of $\mathcal{V}$ so that for all $1 \leq i \leq n, t_{0}$ is a special weak near-unanimity operation for $\mathbf{A}_{i}$ and for all $m \geq 1, \mathbf{A}_{i}=\operatorname{TS}\left(m k_{0}+1\right)$.

Lemma 7.1. A is a simple non-Abelian algebra.

Proof. First we prove that $\mathbf{A}$ is simple. Clearly $|\mathbf{A}|>1$. Suppose that $\mathbf{A}$ has a congruence $\theta$ different from $A \times A$ and the identity relation. We shall derive a contradiction by showing that $\mathbf{A}=\mathrm{TS}\left(k_{0}+1\right)$.

Let $T$ be any non-empty totally symmetric subalgebra of $\mathbf{A}^{k_{0}+1}$. Where $\pi: \mathbf{A} \rightarrow$ $\mathbf{A} / \theta$ is the canonical epimorphism, we have that $\pi^{k_{0}+1}(T)$ is a non-empty totally symmetric subalgebra $T_{\theta}$ of $(\mathbf{A} / \theta)^{k_{0}+1}$. Here $\mathbf{A} / \theta \models \mathrm{TS}\left(k_{0}+1\right)$; thus $T_{\theta}$ has a diagonal vector. This means that there is a vector $\bar{x} \in T$ with the property that for some $\theta$-equivalence class $E, \bar{x} \in E^{k_{0}+1}$. Now $E$ is a subalgebra of $\mathbf{A}$ (since $\mathbf{A}$ is idempotent) and $T \cap E^{k_{0}+1}$ is a non-empty totally symmetric subalgebra of $E^{k_{0}+1}$. Since $\theta \neq A \times A$, then $|E|<|A|$. The algebra $E$ thus satisfies $\operatorname{TS}\left(k_{0}+1\right)$, implying that $T \cap E^{k_{0}+1}$ contains a diagonal vector.

We have shown that every non-empty totally symmetric subalgebra of $\mathbf{A}^{k_{0}+1}$ contains a diagonal vector. This contradicts our assumption that $\mathbf{A}$ satisfies $\operatorname{TS}(k)$ for no $k>1$. The contradiction concludes our proof that $\mathbf{A}$ is simple.

Now suppose that $\mathbf{A}$ is Abelian. Then, as we saw in Section 2, since $\mathbf{A} \in \mathcal{V}$ and $\mathcal{V}$ omits type 1, then $\mathbf{A} \models \mathrm{WNU}(k)$ for some $k>1$ and as we have seen, it follows that $\mathbf{A} \models \operatorname{TS}(k)$ for some $k>1$. This is another contradiction.

Recall that the property $\operatorname{TST}(m)$ was defined in Definition 6.5. We put $M=$ $\max \left(|\mathbf{A}|, k_{0}+1\right) \cdot k_{0}$.

Lemma 7.2. For $m \geq M$ and for $1 \leq i \leq n, \mathbf{A}_{i}=\operatorname{TST}(m)$.

Proof. Suppose that $R$ is a non-empty totally symmetric subalgebra of $\mathbf{A}_{i}^{m}$ where $m>\left|\mathbf{A}_{i}\right| \cdot k_{0}$ and $m \geq k_{0}^{2}+k_{0}$. We must prove that $R$ contains the vector $c^{m-1} b$ for some $b, c \in A_{i}$.

If $m \equiv 1\left(\bmod k_{0}\right)$ then $\mathbf{A}_{i} \models \operatorname{TS}(m)$ implying that $\mathbf{A}_{i} \models \operatorname{TST}(m)$. There is nothing to prove in this case. In the contrary case, we write $m=\ell k_{0}+1+r$ where $\ell>0$ and $0<r<k_{0}$. Choose any vector $\bar{x} \in R$. Since $m>\left|\mathbf{A}_{i}\right| \cdot k_{0}>\left|\mathbf{A}_{i}\right| \mid c d o t r$, 
some member of $A_{i}$ occurs $r$ times in $\bar{x}$. After a permutation of coordinates in $\bar{x}$, we get, say, $a^{r} \bar{u} \in R$ where $a \in A_{i}$ and $\bar{u} \in A_{i}^{\ell k_{0}+1}$. Thus

$$
S=\left\{\bar{y} \in A_{i}^{\ell k_{0}+1}: a^{r} \bar{y} \in R\right\}
$$

is a non-empty totally symmetric subalgebra of $\mathbf{A}_{i}^{\ell k_{0}+1}$. It follows that there is $b \in A_{i}$ with $a^{r} b^{\ell k_{0}+1} \in R$. Rewrite this as $a^{r} b^{\ell k_{0}} b \in R$ and note that the assumed inequality for $m$ implies that $\ell \geq k_{0}>r$. Now the argument proving Claim 4.11 in the proof of Lemma 4.9 shows that there is $c \in A_{i}$ with $c^{r+\ell k_{0}} b \in R$, i.e, $c^{m-1} b \in R$.

Definition 7.3. An algebra $\mathbf{C}$ satisfies $\operatorname{IST}(k)$ iff for all $a, b \in C$ there exists an element $c \in C$ so that the totally symmetric subalgebras of $\mathbf{C}^{k}$ generated by $a^{k-1} b$ and $a^{k-1} c$ contain the vector $c^{k}$.

Lemma 7.4. Let $\mathbf{C}$ be a finite algebra. If $\mathbf{C}$ satisfies $\mathrm{ST}(k)$, then it satisfies $\operatorname{IST}\left(k^{|\mathbf{C}| !}\right)$.

Proof. Since $\mathbf{C}$ satisfies $\mathrm{ST}(k)$, we can choose, for each pair $(a, b) \in C^{2}$ a term $t_{a, b}$ of arity $k$ so that

$$
t_{a, b}(b, a, \ldots, a)=t_{a, b}(a, b, a, \ldots, a)=\cdots=t_{a, b}(a, \ldots, a, b) .
$$

For each $a \in C$ we define a unary operation $f_{a}$ on $C$ as

$$
f_{a}(b)=t_{a, b}(b, a, \ldots, a) .
$$

Consider the term

$$
s\left(x_{0}, \ldots, x_{k^{2}-1}\right)=t_{a, f_{a}(b)}\left(t_{a, b}\left(x_{0}, \ldots, x_{k-1}\right), \ldots, t_{a, b}\left(x_{k(k-1)}, \ldots, x_{k^{2}-1}\right) .\right.
$$

Since $\mathbf{C}$ is idempotent, $s(b, a, \ldots, a)=\cdots=s(a, \ldots, a, b)=f_{a}\left(f_{a}(b)\right)$. By iterating this argument, for each $a, b \in C$ we get a term $s_{a, b}$ of arity $k^{|C| !}$ so that

$$
s_{a, b}(b, a, \ldots, a)=\cdots=s_{a, b}(a, \ldots, a, b)=f_{a}^{|C| !}(b) .
$$

Put $c=f_{a}^{|C| !}(b)$. We know that the $|C|$ !-th power of the function $f_{a}$ is idempotent; that is $f_{a}^{|C| !}(c)=f_{a}^{|C| !}\left(f_{a}^{|C| !}(b)\right)=f_{a}^{|C| !}(b)=c$. This implies that

$$
s_{a, c}(c, a, \ldots, a)=\cdots=s_{a, c}(a, \ldots, a, c)=f_{a}^{|C| !}(c)=c,
$$

so we have the required terms for $\operatorname{IST}\left(k^{|C| !}\right)$.

Definition 7.5. An algebra $\mathbf{C}$ satisfies $\operatorname{RST}(k)$ iff for every minimal $\star$-subalgebra $T \subseteq C^{k}, D(T) \neq C$.

Lemma 7.6. For $m>M$, we have that A satisfies $\operatorname{RST}(m)$. For $m>M$ and $m \equiv 1\left(\bmod k_{0}\right)$, we have that $\mathbf{A}$ satisfies $\mathrm{ST}(m)$.

Proof. Let $m>M$ and let $T$ be a minimal $\star$-subalgebra of $\mathbf{A}^{m}$. Clearly we have $m \geq 3$. By Lemma 7.2, every proper subalgebra of $\mathbf{A}$ satisfies TST $(m-1)$. Thus it follows from Lemma 7.1 and Theorem 6.7 that $D(T) \neq A$. This argument shows that $\mathbf{A} \models \operatorname{RST}(m)$.

Next, let $m>M, m=\ell k_{0}+1$, and let $T$ be a minimal $\star$-subalgebra of $\mathbf{A}^{m}$. Since $\mathbf{A}=\operatorname{RST}(m)$, then $T$ is actually a minimal $\star$-subalgebra of $\mathbf{B}^{m}$ for some proper subalgebra $\mathbf{B}<\mathbf{A}$. But $\mathbf{B} \cong \mathbf{A}_{i}$ for some $1 \leq i \leq n$ and $\mathbf{B}=\mathrm{ST}(m)$. Thus $T$ is a singleton, and contains a diagonal vector. This shows that $\mathbf{A} \models \mathrm{ST}(m)$. 
Now let $k_{1}$ be the least multiple of $k_{0}$ that is not less than $M$ and not less than $|A|$, and let $k$ be the multiple of $k_{0}$ such that $k+1=\left(k_{1}+1\right)^{|\mathbf{A}| !}$. Let $t$ be a term of $k+1$ variables which is a special weak near-unanimity term for all $\mathbf{A}_{i}, 1 \leq i \leq n$. The proof of Lemma 4.8 , starting with $t_{0}$, will produce such a term.

The next lemma summarizes what we know about $\mathbf{A}$.

Lemma 7.7. Let $\mathbf{A}$ be the algebra from the previous lemma.

(1) A is a finite, simple, non-Abelian algebra that satisfies $\operatorname{TS}(m)$ for no $m>1$.

(2) $t$ is a $k+1$-ary term which is a special weak near-unanimity term for every proper subalgebra of $\mathbf{A}$.

(3) Each proper subalgebra of $\mathbf{A}$ satisfies $\mathrm{TS}(m k+1)$ for all $m \geq 1$.

(4) Each proper subalgebra of $\mathbf{A}$ satisfies $\mathrm{TST}(m)$ for all $m \geq k$.

(5) A satisfies $\operatorname{RST}(m)$ for all $m \geq k$.

(6) A satisfies $\mathrm{ST}(m k+1)$ for all $m>0$.

(7) A satisfies $\operatorname{IST}(k+1)$.

Proof. These statements are easily justified with the help of the preceding lemmas of this section.

The next lemma contradicts statement (1) of the above lemma. Its proof will conclude our proof by contradiction, of Theorem 1.1.

Lemma 7.8. Let $\mathbf{A}$ be the algebra and $k$ be the integer from the previous lemma. Then A satisfies $\operatorname{TS}(N)$ for some large integer $N$.

Proof. Let $N$ be large and $N \equiv 1 \bmod k$, and let $\mathbf{B} \leq \mathbf{A}^{N}$ be an arbitrary totally symmetric subalgebra. We argue that $\mathbf{B}$ contains a diagonal element.

Claim 7.9. Suppose that $a^{i} b^{j k} \bar{x} \in B$ for some integers $1 \leq i \leq j$ and $a, b, \bar{x}$. Then $c^{i+j k} \bar{x} \in B$ for some $c \in A$.

Use an analogous argument to that of the proof of the first two claims in our proof of Lemma 4.9, using the fact that $\mathbf{A}$ satisfies $\operatorname{IST}(k+1)$ as a replacement for the existence of a special weak near-unanimity term for $\mathbf{A}$.

Claim 7.10. If $a_{1}^{k} \ldots a_{p}^{k} \bar{x} \in B$ and $p>k|A|$, then $b^{p k} \bar{x} \in B$ for some $b \in A$.

Let $i \leq p$ be a maximal integer so that $b^{i k} c_{1}^{k} \ldots c_{p-i}^{k} \bar{x} \in B$ for some elements $b, c_{1}, \ldots, c_{p-i} \in A$. By the pigeon-hole principle $i \geq k$. If $i<p$, then we can use Claim 7.9 for $b^{i k} c_{1}^{k}$ to get that $d^{i k+k} c_{2}^{k} \ldots c_{p-i}^{k} \bar{x} \in B$. This contradiction shows that $i=p$.

Claim 7.11. $a^{i} b^{N-i-1} c \in B$ for some $a, b, c \in A$ and integer $i<k$.

Take an arbitrary tuple from $B$ and write it in the form $a_{1}^{k} \ldots a_{p}^{k} b^{q} c_{1} \ldots c_{r}$. Choose such a tuple where $k p+q$ is maximal and $0 \leq q<k$. If $q=0$, then $r=0$ by the maximality of $k p+q$. If $r=0$, then the claim holds by Claim 7.10. (Here we require only that $N-1>k^{2}|A|$.) Therefore, we may assume that $q>0$ and $r>0$.

Clearly, $r<k|A|$, otherwise $k p+q$ was not maximal by the pigeon-hole principle. Thus by Claim 7.10 (assuming that $N>\left(k^{2}+k\right)|A|+k$ ), we may assume that $a_{1}=$ $\cdots=a_{p}$. Using Claim 7.9 for $c_{1}$ and $a_{1}^{p k}$ we get a tuple of the form $d^{p k} b^{q} d c_{2} \ldots c_{r}$. If $\left\{d, c_{2}, \ldots, c_{r}\right\}$ generates $\mathbf{A}$ as a subalgebra, then $k p+q$ was not maximal, because the totally symmetric subalgebra of $A^{r}$ generated by $d c_{2} \ldots c_{r}$ contains a tuple of 
the form $b c_{2}^{\prime} \ldots c_{r-1}^{\prime}$. Now we use $\operatorname{TST}(N-q)$ for the coordinates where we have elements from $\left\{d, c_{2}, \ldots, c_{r}\right\}$, which yields a tuple of the form $b^{q} e^{N-q-1} f$.

ClaIm 7.12. $a^{N-2} b c \in B$ for some $a, b, c \in A$.

From the previous claim we know that $a^{i} b^{N-i-1} c \in B$ for some elements $a, b, c \in$ $A$ and integer $i<k$. Since A satisfies $\operatorname{RST}(k)$ we can apply this to $a b^{k-1}$ to get $d e^{k-1}$ such that $d, e$ generate a proper subalgebra of $\mathbf{A}$. We do this $i$-many times, once to each $a$, and get that $d^{i} e^{k i-i} b^{N-1-k i} c \in B$. As $d, e$ generate a proper subalgebra, we can apply $T S T(k i)$ and get that $f g^{k i-1} b^{N-1-k i} c \in B$ for some elements $f, g \in A$. Since $N \equiv 1(\bmod k), N-1-k i \equiv 0(\bmod k)$. Moreover, by chosing $N$ large enough, we can make $N-1-k i$ arbitrary large. Therefore, we can apply Claim 7.9 to the tuple $g^{k i-1} b^{N-1-k i}$, and get that $f h^{N-2} c \in B$.

Claim 7.13. $a b^{i} c^{N-i-1} \in B$ for some $a, b, c \in A$ and integer $i \leq|A|$ such that $\{a, c\}$ and $\{b, c\}$ generate proper subalgebras of $\mathbf{A}$.

From Claim 7.12 we have a tuple $a b c^{N-2} \in B$. By $\operatorname{RST}(N-1)$ we can assume that $\{b, c\}$ generates a proper subalgebra of $\mathbf{A}$. We define a sequence $a_{1}, a_{2}, \cdots \in A$ of elements such that $a_{i} b^{i} c^{N-i-1} \in B$. Put $a_{1}=a$. If $a_{i}$ is defined and $\left\{a_{i}, c\right\}$ generates $\mathbf{A}$, then there is a binary term $t$ such that $t\left(a_{i}, c\right)=b$. In this case we put $a_{i+1}=t\left(c, a_{i}\right)$. By the construction we have that $a_{i+1} b^{i+1} c^{N-i-1} \in B$. If for some $i \leq|A|$ we arrive to an element $a_{i}$ so that $\left\{a_{i}, c\right\}$ does not generate $\mathbf{A}$ then we are done. Otherwise, we have a repetition in $a_{1}, \ldots, a_{|A|}, a_{|A|+1}$, so we can continue with the repeated sequence to get that $a_{N-1} b^{N-1} \in B$. From this by $\operatorname{ST}(N)$ we get a constant tuple.

Claim 7.14. $a b_{0}^{k} \ldots b_{k}^{k} c^{N-1-k-k^{2}} \in B$ for some $a, b_{1}, \ldots, b_{k+1}, c \in A$.

From the previous claim we have a tuple $a b^{i} c^{N-i-1} \in B$. It is easy to see that we have $k>|A| \geq i$. Thus we can permute the coordinates to get

$$
c^{k^{2}} b_{0} \cdots b_{k} c^{N-k^{2}-k-1} \in B
$$

where $b_{0} \cdots b_{k}=a b^{i} c^{k-i}$. Now $\left\{b_{i}, c\right\}$ generates a proper subalgebra of $\mathbf{A}$ for $0 \leq i \leq k$; hence $t$ satisfies the weak-NU equations on inputs from $\left\{b_{i}, c\right\}$ for each $i$. Now the argument used to prove Claim 4.12 in the proof of Lemma 4.9 works in this situation, using the term $t$; and it yields the desired result.

Claim 7.15. B contains a diagonal element.

Observe, that $N-1-k-k^{2} \equiv 0(\bmod k)$, so we can apply Claim 7.10 to the tuple $a b_{0}^{k} \ldots b_{k}^{k} c^{N-1-k-k^{2}} \in B$ and get that $a d^{N-1} \in B$ for some $d \in A$. Now using $\mathrm{ST}(N)$ we get a diagonal tuple in $B$.

\section{Proof of Theorem 1.2}

D. Hobby, R. McKenzie [6] [Theorem 9.10] gives the equivalence of statements (1) and (2) in Theorem 1.2. We showed in Section 2 that (4) implies (1), and (3) trivially implies (4). The proof of Theorem 1.2 will therefore be completed with the proof of the next theorem, which shows that (1) implies (3).

Theorem 8.1. Let $\mathcal{V}$ be a locally finite variety that omits types $\mathbf{1}$ and $\mathbf{2}$. Let $\mathbf{A}$ be any finite algebra in $\mathcal{V}$ with $n=|\mathbf{A}|>1$. For $k>2(n-1)$ !, $\mathbf{A}$ satisfies $\operatorname{ST}(k)$, and for $k \geq 2 n$ !, A satisfies $\operatorname{TS}(k)$ and $\mathrm{WNU}(k)$. 
Proof. First, note that since $\mathcal{V}$ omits types $\mathbf{1}$ and $\mathbf{2}$, it has no Abelian algebra with more than one element.

We prove this theorem by induction on $n$. If $\mathbf{A} \in \mathcal{V}$ and $n=|\mathbf{A}|=2$ then $\mathbf{A}$ is simple and non-Abelian. Let $k>2(=2(n-1)$ !). Now, proper subalgebras of $\mathbf{A}$ have only one element and thus satisfy $\operatorname{TS}(k)$, and $\operatorname{TST}(k)$. Thus it is immediate from Theorem 6.7 that every minimal $\star$-subalgebra $T$ of $\mathbf{A}^{k}$ has $D(T) \neq A$, implying that $T$ is a singleton. This means that $\mathbf{A}$ satisfies $\operatorname{ST}(k)$. To conclude the proof for $n=2$, notice that since $\mathbf{A} \models \mathrm{ST}(k)$ for $k \geq 3$, then by Theorem $4.17, \mathbf{A} \models \operatorname{TS}(k)$ for $k \geq 4=2 n$ !, and then by Lemma $4.5, \mathbf{A}=\mathrm{WNU}(k)$ for $k \geq 4$ as well.

Now suppose that $n>2$ and the conclusions of this theorem are valid for all finite algebras $\mathbf{A} \in \mathcal{V}$ with $2 \leq|\mathbf{A}|<n$. Let $\mathbf{A} \in \mathcal{V}$ with $|\mathbf{A}|=n$, and let $k>2(n-1)$ !. By our induction assumption, every proper subalgebra and every proper homomorphic image of $\mathbf{A}$ satisfies $\operatorname{TS}(k)$ and $\operatorname{TS}(k-1)$, and thus also satisfies $\operatorname{TST}(k-1)$. Thus the same proof used in the proof of Lemma 7.1 gives us the conclusion that $\mathbf{A}$ satisfies $\mathrm{ST}(k)$, unless $\mathbf{A}$ is simple. Suppose that $\mathbf{A}$ is simple. Let $T$ be any minimal $\star$-subalgebra of $\mathbf{A}^{k}$. We have that all the hypotheses of Theorem 6.7 except possibly the hypothesis that $D(T)=A$ are true. The conclusion of Theorem 6.7 is false (as $\mathbf{A}$ is non-Abelian). Thus we are forced to the conclusion that $D(T) \neq A$. In this case, $T$ is a minimal $\star$-subalgebra of $\mathbf{B}^{k}$, $B=D(T)$, and $\mathbf{B}=\mathrm{ST}(k)$. Thus $T$ is a singleton. So we conclude that $\mathbf{A}=\mathrm{ST}(k)$. This holds for all $k \geq m=2(n-1) !+1$. By Theorem 4.17 , we have that $\mathbf{A} \models \operatorname{TS}(k)$ for all $k \geq(m-1)|A|=2 n$ !. By Lemma $4.5, \mathbf{A} \models \mathrm{WNU}(k)$ for the same values of $k$. This completes our inductive proof of Theorem 8.1.

The proof of Theorem 1.2 is now complete.

\section{Proof of Theorem 1.3}

By a system of Jónsson operations on $A$ we mean a sequence $d_{1}(x, y, z), \ldots$, $d_{2 n-1}(x, y, z)$ of operations (for some $n \geq 1$ ) satisfying the equations

$$
\begin{aligned}
x & \approx d_{j}(x, y, x) \text { when } 1 \leq j \leq 2 n-1, \\
x & \approx d_{1}(x, z, z), \\
d_{2 i}(x, z, z) & \approx d_{2 i+1}(x, z, z) \text { for all } 1 \leq i \leq n-1, \\
d_{2 i-1}(x, x, z) & \approx d_{2 i}(x, x, z) \text { for all } 1 \leq i \leq n-1, \\
d_{2 n-1}(x, x, z) & \approx z .
\end{aligned}
$$

According to a classical result of B. Jónsson [8], a variety $\mathcal{V}$ is congruence distributive iff $\mathcal{V}$ has terms $d_{1}(x, y, z), \ldots, d_{2 n-1}(x, y, z)$ such that the above equations are valid in $\mathcal{V}$, for some $n \geq 1$. Such a sequence of terms is called a sequence of Jónsson terms for $\mathcal{V}$.

Definition 9.1. Suppose that $\mathbf{A}$ is an algebra and $m>2$, and $\mathbf{B}$ is a subalgebra of $\mathbf{A}^{m}$. For $a, b, c \in A$ we define a condition

$C_{B}(a, b, c)$ : for all $0 \leq i \leq m-1$, the $m$-tuple $b c^{(i)} a^{(m-i-1)}$ belongs to $B$.

Let $f(x, y, z)$ be a term operation of $\mathbf{A}$. We define three conditions on $f$ (actually, on $(\mathbf{B}, \mathbf{A}, f))$ :

$\left(C_{f}^{1}\right)$ There exist $a, b \in A$ so that, where $c=f(a, a, b)$, the condition $C_{B}(a, b, c)$ holds. 
$\left(C_{f}^{2}\right)$ There exist $a, b \in A$ so that, where $d=f(a, b, b)$, the condition $C_{B}(a, b, d)$ holds.

$\left(C_{f}\right)$ There exist $a, b \in A$ so that, where $c=f(a, a, b)$ and $d=f(a, b, b)$, the conditions $C_{B}(a, b, c)$ and $C_{B}(a, b, d)$ hold.

Lemma 9.2. Let $\mathbf{A}=\langle A, f\rangle$ be an algebra with one ternary operation such that $\mathbf{A}=f(x, y, x) \approx x$. Let $m$ be an integer greater than 2 . Let $\mathbf{B}$ be a totally symmetric subalgebra of $\mathbf{A}^{m}$. The conditions $\left(C_{f}^{1}\right),\left(C_{f}^{2}\right)$ and $\left(C_{f}\right)$ are equivalent.

Proof. We assume that $C_{B}(a, b, c)$ holds, and either $c=f(a, a, b)$ or $c=f(a, b, b)$. We proceed to construct $a^{\prime}, b^{\prime} \in A$ and show that $C_{B}\left(a^{\prime}, b^{\prime}, f\left(a^{\prime}, a^{\prime}, b^{\prime}\right)\right)$ and $C_{B}\left(a^{\prime}, b^{\prime}, f\left(a^{\prime}, b^{\prime}, b^{\prime}\right)\right)$ hold. We begin by defining $b_{0}=b$ and for $0 \leq i<m-1$, $b_{i+1}=f\left(b_{i}, c, c\right)$.

Claim 1: For all $0 \leq i \leq j \leq m-1$ we have $b_{i} c^{(j)} a^{(m-j-1)} \in B$. We prove this claim by induction on $i$. For $i=0$, the claim is equivalent to condition $C_{B}(a, b, c)$ which we are assuming. Now suppose that we have a certain $i, 0 \leq i<m-1$, and $b_{i} c^{(j)} a^{(m-j-1)} \in B$ for all $i \leq j \leq m-1$. Let $i+1 \leq j \leq m-1$. To see that $b_{i+1} c^{(j)} a^{(m-j-1)} \in B$, we apply $f^{\mathbf{B}}$ to some members of $\mathbf{B}$ below. In this calculation, we write members of $\mathbf{B}$ ( $m$-tuples) as column vectors. The operation $f$ is being applied across the rows of an $m \times 3$ matrix.

$$
\left[\begin{array}{c}
b_{i+1} \\
c \\
c \\
c \\
\cdot \\
\cdot \\
c \\
a \\
\cdot \\
\cdot \\
a
\end{array}\right]=f\left[\begin{array}{ccc}
b_{i} & c & c \\
a & a & b \\
c & b & c \\
c & a & c \\
\cdot & & \\
\cdot & & \\
c & a & c \\
a & a & a \\
\cdot & & \\
\cdot & & \\
a & a & a
\end{array}\right] \text { or }\left[\begin{array}{c}
b_{i+1} \\
c \\
c \\
c \\
\cdot \\
\cdot \\
c \\
a \\
\cdot \\
\cdot \\
a
\end{array}\right]=f\left[\begin{array}{ccc}
b_{i} & c & c \\
a & b & b \\
c & a & c \\
c & a & c \\
\cdot & & \\
\cdot & & \\
c & a & c \\
a & a & a \\
\cdot & & \\
\cdot & & \\
a & a & a
\end{array}\right]
$$

The first formula applies if $c=f(a, a, b)$; the second applies if $c=f(a, b, b)$. The third row of the $m \times 3$ matrix in the first formula is $c b c$ if $j \geq 2$, and is $a b a$ if $j=1(=i+1)$. The first and third columns of each matrix contain $c$ in positions 3 through $j+2$. In both cases, all three columns of the matrix denote elements of $B$ (due to the induction assumption). Hence $b_{i+1} c^{(j)} a^{(m-j-1)} \in B$ as required.

Claim 2: Where $b^{\prime}=b_{m-1}, c^{\prime}=f\left(c, c, b^{\prime}\right), d^{\prime}=f\left(c, b^{\prime}, b^{\prime}\right)$ we have for all $0 \leq$ $j \leq i \leq m-1$, that $b_{i} c^{(j)} c^{(m-j-1)} \in B, b_{i} d^{\prime(j)} c^{(m-j-1)} \in B$.

This claim is proved by induction on $j$. The first claim justifies this claim for $j=0$. Next, let $j=1$ and let $i$ be fixed, $1 \leq i \leq m-1$. Note that by Claim $1, b^{\prime} c^{(m-1)} \in b$, and symmetrically, $c b^{\prime} c^{(m-2)} \in B$. Then the formulas below show 
that $b_{i} c^{\prime} c^{(m-2)} \in B$ and $b_{i} d^{\prime} c^{(m-2)} \in B$.

$$
\left[\begin{array}{c}
b_{i} \\
c^{\prime} \\
c \\
c \\
\cdot \\
\cdot \\
c
\end{array}\right]=f\left[\begin{array}{ccc}
b_{i-1} & c & c \\
c & c & b^{\prime} \\
c & b & c \\
c & c & c \\
\cdot & & \\
\cdot & & \\
c & c & c
\end{array}\right] \text { and }\left[\begin{array}{c}
b_{i} \\
d^{\prime} \\
c \\
c \\
\cdot \\
\cdot \\
c
\end{array}\right]=f\left[\begin{array}{ccc}
b_{i-1} & c & c \\
c & b^{\prime} & b^{\prime} \\
c & c & c \\
c & c & c \\
\cdot & & \\
\cdot & & \\
c & c & c
\end{array}\right]
$$

Now suppose that $1 \leq j<m-1$ and the claim is true for this $j$ and all $m-1 \geq i \geq j$. Choose any $m-1 \geq i \geq j+1$. To demonstrate that $b_{i} c^{\prime(j+1)} c^{(m-j-2)} \in B$ and $b_{i} d^{\prime(j+1)} c^{(m-j-2)} \in \bar{B}$, we use these formulas:

$$
\left[\begin{array}{c}
b_{i} \\
c^{\prime} \\
c^{\prime} \\
c^{\prime} \\
\cdot \\
\cdot \\
c^{\prime} \\
c \\
\cdot \\
\cdot \\
c
\end{array}\right]=f\left[\begin{array}{ccc}
b_{i-1} & c & c \\
c & c & b^{\prime} \\
c^{\prime} & b & c^{\prime} \\
c^{\prime} & c & c^{\prime} \\
\cdot & & \\
\cdot & & \\
c^{\prime} & c & c^{\prime} \\
c & c & c \\
\cdot & & \\
\cdot & & \\
c & c & c
\end{array}\right] \text { and }\left[\begin{array}{c}
b_{i} \\
d^{\prime} \\
d^{\prime} \\
d^{\prime} \\
\cdot \\
\cdot \\
d^{\prime} \\
c \\
\cdot \\
\cdot \\
c
\end{array}\right]=f\left[\begin{array}{ccc}
b_{i-1} & c & c \\
c & b^{\prime} & b^{\prime} \\
d^{\prime} & c & d^{\prime} \\
d^{\prime} & c & d^{\prime} \\
\cdot & & \\
\cdot & & \\
d^{\prime} & c & d^{\prime} \\
c & c & c \\
\cdot & & \\
\cdot & & \\
c & c & c
\end{array}\right]
$$

In the first $m \times 3$ matrix, the first and third column have $j$ occurences of $c^{\prime}$-likewise in the second formula, the first and third column have $j$ occurences of $d^{\prime}$.

To conclude our proof, we take $a^{\prime}=c, b^{\prime}=b_{m-1}$. Claim 2 with $i=m-1$ gives that both conditions $C_{B}\left(a^{\prime}, b^{\prime}, f\left(a^{\prime}, a^{\prime}, b^{\prime}\right)\right)$ and $C_{B}\left(a^{\prime}, b^{\prime}, f\left(a^{\prime}, b^{\prime}, b^{\prime}\right)\right)$ hold.

To prove Theorem 1.3, we can assume that $\mathbf{A}$ is the free algebra over the Jónsson equations for $d_{1}, \ldots, d_{2 n-1}$, freely generated by $x, y$. The algebra $\mathbf{A}$ is idempotent. Let $m \geq 3$. Put $B=\Delta_{m}$, the totally symmetric algebra generated by $y x x \cdots x$, $x y x \cdots x, \ldots, x x x \cdots x y$. The condition $\left(C_{d_{1}}^{2}\right)$ is true, since $d_{1}(x, y, y)=x$ and $y x x \cdots x \in B$. Notice that for $i$ odd, $\left(C_{d_{i}}^{1}\right)$ is equivalent to $\left(C_{d_{i+1}}^{1}\right)$ since $d_{i}(x, x, y) \approx$ $d_{i+1}(x, x, y)$ is an identity of $\mathbf{A}$; and for $i$ even, $\left(C_{d_{i}}^{2}\right)$ is equivalent to $\left(C_{d_{i+1}}^{2}\right)$. Thus using Lemma 9.2, we deduce that $\left(C_{d_{2 n-1}}^{1}\right)$ must be true. Hence there exists $a, b \in A$ so that, in particular, $b v v \cdots v \in B$, where $v=d_{2 n-1}(a, a, b)$. But here $v=b$ from the last Jónsson equation. Thus $B=\Delta_{m}$ contains a diagonal element $b b \cdots b$. Since the operations are idempotent, this gives an $m$-ary weak near-unanimity operation. 


\section{LOCALLY FINITE CONGRUENCE MODULAR VARIETIES}

A sequence $d_{1}(x, y, z), \ldots, d_{2 n}(x, y, z), p(x, y, z)$ of operations satisfying the equations

$$
\begin{aligned}
x & \approx d_{1}(x, y, y) \\
x & \approx d_{j}(x, y, x) \text { for } 1 \leq j \leq 2 n \\
d_{2 i-1}(x, x, y) & \approx d_{2 i}(x, x, y) \text { for } 1 \leq i \leq n \\
d_{2 i}(x, y, y) & \approx d_{2 i+1}(x, y, y) \text { for } 1 \leq i<n \\
d_{2 n}(x, y, y) & \approx p(x, y, y) \\
p(x, x, y) & \approx y
\end{aligned}
$$

is called a system of Gumm operations on A. H. P. Gumm [5] proved that a variety $\mathcal{V}$ is congruence modular iff $\mathcal{V}$ has terms $d_{1}(x, y, z), \ldots, d_{2 n}(x, y, z), p(x, y, z)$ such that the above equations are valid in $\mathcal{V}$, for some $n \geq 1$. Such a sequence of terms is called a sequence of Gumm terms for $\mathcal{V}$.

Theorem 10.1. Let $\mathbf{A}$ be a finite algebra and $k \geq 3$, and let $\mathbf{T}$ be a minimal $\star$-subalgebra of $\mathbf{A}^{k}$. If $\mathbf{A}$ has Gumm terms then $\mathbf{T}$ is $k-1$-complete.

Proof. Suppose that A has Gumm terms $d_{1}, \ldots, d_{2 n}, p$. It follows from Lemma 9.2 that there are $a, b \in D(T)$ with $b c^{(i)} a^{(k-i-1)} \in T$ for all $0 \leq i \leq k-1$, where $c=d_{2 n}(a, b, b)=p(a, b, b)$. Applying $p$ to the vectors $b a c^{(k-2)}, a b c^{(k-2)}, a b c^{(k-2)}$, we get that $d c^{(k-1)} \in T$ where $d=p(b, a, a)$. Thus $d$ is a co-generic element with respect to $\mathbf{T}$. Then applying $p$ to the vectors $b a^{(k-1)}, a a b a^{(k-3)}, a b a^{(k-2)}$ we get that $d b e a^{(k-3)} \in T$ where $e=p(a, b, a)$. Since $b$ is co-generic, then by Lemma 5.2, $d$ is both generic and co-generic. Thus by the same lemma, $\mathbf{T}$ is $k-1$-complete.

Theorem 10.2. Let $\mathcal{V}$ be a locally finite congruence modular variety. Let $\mathbf{F}$ be the free algebra freely generated by $x, y$ in $\mathcal{V}$, and put $f=|F|$. If $k$ is an integer such that $k \geq 3$ and $k \equiv 1(\bmod f !)$, then $\mathcal{V}$ satisfies $\operatorname{WNU}(k)$.

Proof. We define $\Delta_{k}$ as the subalgebra of $\mathbf{F}^{k}$ generated by the vectors $y x^{k-1}$, $x y x^{k-2}, \ldots, x^{k-1} y$ and we take $\mathbf{T}$ to be a minimal $\star$-subalgebra of $\Delta_{k}$. we must show that $|T|=1$. The Gumm terms are $d_{1}, \ldots, d_{2 n}, p$. By Theorem 10.1, $\mathbf{T}$ is $k-1-$ complete. As in the proof of Theorem 10.1, we have $a, b \in W$ with $b a^{(i)} c^{(k-i-1)} \in T$ for all $0 \leq i \leq k-1$, where $c=p(a, b, b)$.

By $k$-1-completeness, for all $0 \leq i \leq k-1$, there is $x_{i} \in D(T)$ with $x_{i} b^{i} a^{k-i-1} \in$ $T$. Clearly, there are $i \geq 0$ and $j>0$ so that $i+j \leq f<k$ and $x_{i}=x_{i+j}$. Then applying a permutation to the coordinates in the vectors $x_{i} b^{i} a^{k-i-1}$ and $x_{i} b^{i+j} a^{k-i-i-1}$ yields two vectors $a^{(j)} \bar{u}, b^{(j)} \bar{u}$ in $T$ for a certain $j, 0<j \leq f=|F|$.

Now if $\bar{x}=b^{(j)} \bar{v}$ is any vector in $T$ whose first $j$ components are $b$, then $p$ applied to $a^{(j)} \bar{u}, b^{(j)} \bar{u}, b^{(j)} \bar{v}$ is the vector $c^{(j)} \bar{v}$. Thus, by symmetry, any $j b$ 's in a vector belonging to $T$ may be replaced by $c$ 's, yielding a new vector in $T$. Since $b$ is a generic element, and $j$ divides $k-1$, then there is $q \in W$ with $q b^{(k-1)} \in T$ and then, replacing $j b$ 's at a time, we get also, $q c^{(k-1)} \in T$. Recall that we also have $b c^{(k-1)}$ in $T$. Finally, the vectors $\bar{r}_{1}=q b^{(k-1)}, \bar{r}_{2}=b q b^{(k-2)}, \ldots, \bar{r}_{k}=b^{(k-1)} q$ generate $T$. Choose a term $t$ so that

$$
t\left(\bar{r}_{1}, \ldots, \bar{r}_{k}\right)=c^{(k-1)} b .
$$


Thus $t(q, b, \ldots, b)=c$. Now replace $\bar{r}_{1}$ by $q c^{(k-1)}$ and each of $\bar{r}_{2}, \ldots, \bar{r}_{k}$ by $b c^{(k-1)}$ in the displayed equation. This yields the vector $c c^{(k-1)} \in T$. This ends our proof.

\section{REFERENCES}

[1] A.A. Bulatov, A. Krokhin, P.G. Jeavons, Constraint satisfaction problems and finite algebras, in Proceedings of the 27th International Colloquium on Automata, Languages and Programming-ICALP'00, volume 1853 of Lecture Notes in Computer Science, pp. 272-282, Springer-Verlag, 2000.

[2] A.A. Bulatov, P.G. Jeavons, Algebraic structures in combinatorial problems (manuscript).

[3] V. Dalmau, Generalized majority-minority operations are tractable, Logical Methods in Comp. Sci. 2 (4:1), 2006, 1-15.

[4] R. Freese and R. McKenzie, Commutator theory for congruence modular varieties, Cambridge University Press New York, 1987.

[5] H.P. Gumm, Geometrical Methods in Congruence Modular Algebras, Memoirs of the American Mathematical Society, No. 286, 1983.

[6] D. Hobby, R. McKenzie, The Structure of Finite Algebras, Contemporary Mathematics series of the AMS, No. 76, 1988.

[7] P.G. Jeavons, On the algebraic structure of combinatorial problems, Theoretical Computer Science 200 (1998), 185-204

[8] B. Jónsson, Algebras whose congruence lattices are distributive, Math. Scand. 21 (1967), $110-121$.

[9] E. Kiss and M. Valeriote, On tractability and congruence distributivity, Logical Methods in Comp. Sci. 3 (2:6), 2007, 1-20.

[10] B. Larose, L. Zádori, Bounded width problems and algebras, Algebra Universalis, 56 (2007), $439-466$.

Bolyai Institute, University of Szeged, Hungary

E-mail address: mmaroti@math.u-szeged.hu

Department of Mathematics, Vanderbilt University, Nashville, U.S.A.

E-mail address: mckenzie@math.vanderbilt.edu 